\title{
The general-covariant and gauge-invariant theory of quantum particles in classical backgrounds
}

\author{
Hrvoje Nikolić \\ Theoretical Physics Division, Rudjer Bošković Institute, \\ P.O.B. 180, HR-10002 Zagreb, Croatia \\ hrvoje@faust.irb.hr
}

November 11, 2018

\begin{abstract}
A new approach to the concept of particles and their production in quantum field theory is developed. A local operator describing the current of particle density is constructed for scalar and spinor fields in arbitrary gravitational and electromagnetic backgrounds. This enables one to describe particles in a local, general-covariant and gauge-invariant way. However, the current depends on the choice of a 2-point function. There is a choice that leads to the local non-conservation of the current in a gravitational or an electromagnetic background, which describes local particle production consistent with the usual global description based on the Bogoliubov transformation. The most natural choice based on the Green function calculated using the Schwinger-DeWitt method leads to the local conservation of the current, provided that interactions with quantum fields are absent. Interactions with quantum fields lead to the local non-conservation of the current which describes local particle production consistent with the usual global description based on the interaction picture.
\end{abstract}

\section{Introduction}

One of the main problems regarding quantum field theory in curved spacetime is how to introduce the concept of particles. The lack of a natural choice of the time coordinate implies that the generalization of the conventional definition of particles in Minkowski spacetime is not unique in general spacetime [1, 2]. There are three main approaches to coping with this problem. Each of them has some advantages and disadvantages. Let us shortly describe them, emphasizing their disadvantages.

The first approach is based on the theoretical point of view that the concept of particles has no fundamental meaning in field theory [3]. Therefore, one should study only local, well-defined covariant operators, such as $T_{\mu \nu}(x)$, and try to express all observable quantities in terms of these. A disadvantage of such an approach is the fact that particles are what we observe in experiments. If we require that quantum field theory describes the observed objects, then it should describe particles.

The second approach, complementary to the first one, resides on the physical point of view that particles are what particle detectors measure. Therefore, one avoids introducing a particlenumber operator by studying the response of a model of a particle detector. These models 
are usually of the Unruh-DeWitt type [4, 5]. However, such an approach is unable to answer the following questions. If all observables in quantum mechanics are represented by hermitian operators and do not require a model of a corresponding detector, then why is the particle number an exception? Do Unruh-DeWitt type detectors describe the essential properties of real particle detectors (such as a Wilson chamber or a Geiger-Müller counter) in real experiments?

The third approach, perhaps the most popular one, is somewhere between the first two approaches. Although there may not exist a natural choice of the time coordinate in the whole spacetime, some regions of spacetime may have a natural choice of the time coordinate. Accordingly, one introduces different definitions of the particle-number operator for different regions. The two definitions are related by a non-trivial Bogoliubov transformation, which is interpreted as particle production [2, 6, 7] or as a variant of the Unruh effect [4, 8, 9]. In this way, one is able to talk about the average number of particles in some large region of spacetime. However, the average number of particles should make sense in any region of spacetime, especially if the wavelength of the particles is much smaller than the region. The third approach, based on the Bogoliubov transformation, is not local and general-covariant, so it is unable to answer questions such as the following. How to calculate, at least in principle, the average number of particles in an arbitrary region of spacetime? Is the average number of particles in the union of two non-intersecting 3-dimensional regions that lie on the same 3-dimensional spacelike hypersurface equal to the sum of the average number of particles in separate regions? If black holes radiate particles as predicted by Hawking [f], then what is the average particle distribution at, for example, 2 Schwarzschild radii away from the black-hole centre?

There is a lot of similarity between the particle production by a background gravitational field and that by a background electromagnetic field [10, 11, 12]. In particular, just as the former has problems with general covariance, the description of the later through the Bogoliubov transformation has problems with gauge invariance [13]. This is related to the fact that gauge invariance is a local property, while the Bogoliubov-transformation method is based on a global definition of particles.

Recently, a new approach to the concept of particles in quantum field theory has been proposed [14]. In this paper we further develop the ideas introduced in [14 and show that the new approach avoids the disadvantages of the other existing approaches discussed above. This new approach is inspired by some advantages of the first and the third approach and does not rely on any model of a particle detector. The main idea is the assumption that there should exist a well-defined local operator of particle-number density. There are two reasons for such an assumption. First, this allows a general-covariant and gauge-invariant formulation. Second, although the particle-number operator is a global operator in the conventional approach, this assumption is consistent with the fact that particles appear as local objects in all existing experiments.

Our approach is based on a similarity between the number of particles and charge. For complex fields, the total number of particles is the sum of the number of particles and antiparticles, while the total charge is the difference of these two numbers. The concept of charge can be described in a local and covariant manner because there exists a local vector current of charge density. We find that a similar vector current exists for the number of particles as well. Nevertheless, it appears that this local current is not unique, but depends on the choice of a 2-point function. When a unique (or a preferred) vacuum exists, then the 2-point function is equal to the Wightman function and the current is conserved. When such a vacuum does not exist, then there is a choice of the 2-point function that leads to the local non-conservation of the current in a gravitational or electromagnetic background, which describes local particle production consistent with the usual global description based on the Bogoliubov transformation. Another choice, based on the Green function calculated using the Schwinger-DeWitt method 
[15, 16], seems to be more natural. This choice leads to the local conservation of the current in an arbitrary gravitational or electromagnetic background, provided that other interactions are absent.

Before studying the new local concept of particles in Secs. 3, 4, and 5, in Sec. 2 we clarify some physical aspects of the concept of particles in the conventional approach. In Sec. 3 we study in detail the case of hermitian scalar fields. In Secs. 4 and 5 we generalize the analysis to complex scalar fields and spin-1/2 fields, respectively. The conclusions and prospects for further investigations are given in Sec. 6. In the Appendix, we derive the particle-density operator in non-relativistic quantum field theory.

\section{Qualitative remarks on the concept of particles}

In quantum field theory, an $n$-particle state is defined as a normalized state of the form

$$
|n\rangle=\int d^{3} k_{1} \cdots d^{3} k_{n} f\left(\mathbf{k}_{1}, \ldots, \mathbf{k}_{n}\right) a_{\mathbf{k}_{1}}^{\dagger} \cdots a_{\mathbf{k}_{n}}^{\dagger}|0\rangle .
$$

The crucial question we attempt to answer in this section is why such formally defined states correspond to the states observed in typical experiments as $n$ separated entities, i.e., "particles".

We introduce the concept of a classical particle detector, such as a Wilson chamber or a Geiger-Müller counter. We call such detectors classical, because, in order to understand how and why such detectors respond, quantum field theory is not essential. Classical detectors respond to states that correspond to the classical concept of particle, i.e., to states which are well localized in space. For example, if two localized particles are very near each other, which can be achieved by a suitable choice of the function $f\left(\mathbf{k}_{1}, \mathbf{k}_{2}\right)$, then a classical detector will see this state as one particle. The response of a classical detector is also localized in time, in the sense that it can, with good precision, determine the instant of time at which the particle takes a certain position in space. In principle, the product of uncertainties $\Delta E \Delta t$ may be arbitrarily small [17]. Because of these local features, one does not expect that the response of a classical detector depends on global aspects such as the whole detector's trajectory or the existence of an event horizon. Therefore, classical detectors are very different from particle detectors of the Unruh-DeWitt type. Because of locality, one expects that classical particle detectors detect objects that are covariant with respect to general coordinate transformations. Therefore, one needs a local and general-covariant notion of a particle in quantum field theory.

As we mentioned above, a many-particle state can be detected as one particle if these localized particles are very near each other. However, it seems that such a situation does not occur in practice. Why? Before answering this question, first consider how a one-particle state

is detected. Assume that a particle detector is localized in space. The function $f\left(\mathbf{k}_{1}\right)$ may correspond to a plane wave or to a state with two lumps, but such states cannot be detected by the localized detector. Such a state will not be detected until the function $f\left(\mathbf{k}_{1}\right)$ collapses to a one-lump state. (We do not attempt to answer the question whether the wave function collapses spontaneously or the detector causes the collapse. In some interpretations of quantum mechanics there is a clear answer to this question, but we do not want to prefer any particular interpretation.)

Now consider a two-particle state. Assume that, a priori, all functions $f\left(\mathbf{k}_{1}, \mathbf{k}_{2}\right)$ are equally probable. (This probability does not reflect a stochastic nature of quantum states, but simply our ignorance of the details that govern the dynamics and the initial conditions.) However, the number of functions corresponding to a two-lump state is much larger than that for a onelump state, so it is very improbable that two particles will form one lump. (Of course, the numbers of two-lump states and one-lump states are both infinite, but their ratio is not equal 
to 1 . To visualize this, note that if there are $N$ places where a lump can be placed, then (for a fixed number of particles) there are $N$ different one-lump states and $N^{2}-N$ different two-lump states.) A four-lump state, for instance, is even more probable than a two-lump state, but if one particle is distributed in more than one lump, it will not be detected.

In this way we have explained why an $n$-particle state behaves as an $n$-lump state in experiments; a particle splited in more than one lump will not be detected, while a smaller number of lumps can be realized in a smaller number of ways, so it has a smaller probability. Of course, if there are strong attractive forces among fields, there may exist a natural tendency for $n$-particle states to form a one-lump state (hadrons, $\alpha$-particles), in which case it is more convenient to treat such states as one-particle states. Actually, it is often completely incorrect to treat such states as $n$-particle states, because non-perturbative effects of interactions may completely change the spectrum of states in a free theory, such as is the case for QCD.

One may argue that classical detectors are not the best operational way to define a particle. One should rather study quantum detectors. (Even the response of a "classical" detector should be ultimately described by quantum field theory.) For example, an atom may be viewed as a quantum photon detector as it absorbs precisely one photon, not a half of it, nor two photons. It is often thought that this is an inherent quantum property. However, it is important to emphasize that there is not any deep, fundamental principle that forbids absorption or emission of a half of a particle or two particles. It is merely a consequence of a particular form of dynamics. For example, an electron in the atom absorbs one photon because the interaction Lagrangian $\mathcal{L}_{\text {int }}=e \bar{\psi} \gamma_{\mu} \psi A^{\mu}$ is linear in $A^{\mu}$. Of course, higher-order corrections allow absorption of two photons (sum of their energies must be equal to the difference of energies of the atom levels), but such processes are suppressed dynamically (small coupling constant) and kinematically (small probability of a one-lump two-photon state). However, is it, in principle, possible to absorb a half of a particle? One can exclude such a possibility by proposing that the Lagrangian $\mathcal{L}\left(\phi, \partial_{\mu} \phi, \ldots\right)$ of fundamental fields should be an analytic function around zero. For example, assuming that there are no derivative couplings, the analyticity implies that a local interaction Lagrangian has a form

$$
\mathcal{L}_{\text {int }}(\phi(x), \cdots)=\sum_{n \geq 0} C_{n}(x)(\phi(x))^{n},
$$

where $n$ are integers and $C_{n}(x)$ depend on some other fields. The interaction (2) implies that the number of absorbed or emitted particles must be an integer. If $\phi(x)$ is an effective, composite field, like $\phi(x)=\chi(x) \chi(x)$, then one can have a term proportional to $\sqrt{\phi}=\chi$, in which case a "half" of the $\phi$-particle, i.e., one $\chi$-particle, can be absorbed or emitted. If one allows terms like $\sqrt{\phi}$ for fundamental fields, then even a "half" of a fundamental particle can be absorbed or emitted. (In this case, the concept of particle based on perturbative calculations is no longer a good concept. In particular, it is not clear, even algebraically, what $\sqrt{a^{\dagger}}|0\rangle$ is.)

\section{Hermitian scalar field}

The hermitian scalar field is the simplest example of a quantum field. Therefore, we start the quantitative analysis by studying this simplest case in detail. The first two subsections do not contain new results, but they are written for the sake of completeness and easier understanding of our later results. 


\subsection{Canonical quantization}

Let $g_{\mu \nu}$ be a classical background metric, $g$ the determinant of $g_{\mu \nu}$, and $R$ the curvature calculated from $g_{\mu \nu}$. The action of a hermitian scalar field $\phi$ is

$$
S=\int d^{4} x|g|^{1 / 2} \frac{1}{2}\left[g^{\mu \nu}\left(\partial_{\mu} \phi\right)\left(\partial_{\nu} \phi\right)-m^{2} \phi^{2}-\xi R \phi^{2}\right],
$$

where $\xi$ is a coupling constant. Writing this as

$$
S=\int d^{4} x|g|^{1 / 2} \mathcal{L}
$$

the canonical-momentum vector is

$$
\pi_{\mu}=\frac{\partial \mathcal{L}}{\partial\left(\partial^{\mu} \phi\right)}=\partial_{\mu} \phi
$$

The correponding equation of motion is

$$
\left(\nabla^{\mu} \partial_{\mu}+m^{2}+\xi R\right) \phi=0
$$

where $\nabla^{\mu}$ is the covariant derivative. Let $\Sigma$ be a spacelike Cauchy hypersurface with a unit vector $n^{\mu}$ normal to $\Sigma$. The canonical-momentum scalar is defined as

$$
\pi=n^{\mu} \pi_{\mu}
$$

The volume element on $\Sigma$ is

$$
d \Sigma^{\mu}=d^{3} x\left|g^{(3)}\right|^{1 / 2} n^{\mu}
$$

The scalar product is defined as

$$
\left(\phi_{1}, \phi_{2}\right)=i \int_{\Sigma} d \Sigma^{\mu} \phi_{1}^{*} \stackrel{\leftrightarrow}{\partial_{\mu}} \phi_{2}
$$

where $a \stackrel{\leftrightarrow}{\partial_{\mu}} b=a \partial_{\mu} b-\left(\partial_{\mu} a\right) b$. If $\phi_{1}$ and $\phi_{2}$ are solutions of (6), then (9) does not depend on $\Sigma$ 18 .

We choose coordinates $(t, \mathbf{x})$ such that $t=$ constant on $\Sigma$. In these coordinates

$$
n^{\mu}=g_{0}^{\mu} / \sqrt{g_{00}},
$$

and the canonical commutation relations are

$$
\begin{gathered}
{\left[\phi(x), \phi\left(x^{\prime}\right)\right]_{\Sigma}=\left[\pi(x), \pi\left(x^{\prime}\right)\right]_{\Sigma}=0,} \\
{\left[\phi(x), \pi\left(x^{\prime}\right)\right]_{\Sigma}=\left|g^{(3)}\right|^{-1 / 2} i \delta^{3}\left(\mathbf{x}-\mathbf{x}^{\prime}\right) .}
\end{gathered}
$$

The label $\Sigma$ denotes that $x$ and $x^{\prime}$ lie on $\Sigma$. Eq. (12) can be written in a manifestly covariant form as

$$
\int_{\Sigma} d \Sigma^{\prime \mu}\left[\phi(x), \partial_{\mu}^{\prime} \phi\left(x^{\prime}\right)\right] \chi\left(x^{\prime}\right)=\int_{\Sigma} d \Sigma^{\prime \mu}\left[\phi\left(x^{\prime}\right), \partial_{\mu} \phi(x)\right] \chi\left(x^{\prime}\right)=i \chi(x),
$$

where $\chi\left(x^{\prime}\right)$ is an arbitrary test function.

For practical calculations, it is more convenient to introduce the quantity $\tilde{n}^{\mu}$ defined as

$$
\tilde{n}^{\mu}=\left|g^{(3)}\right|^{1 / 2} n^{\mu},
$$


where the tilde on $\tilde{n}^{\mu}$ denotes that it is not a vector. It has the property $\nabla_{\mu} \tilde{n}_{\nu}=0$ and has the explicit form

$$
\tilde{n}^{\mu}=\left(\left|g^{(3)}\right|^{1 / 2} / \sqrt{g_{00}}, 0,0,0\right) .
$$

The volume element (8) can be written as

$$
d \Sigma^{\mu}=d^{3} x \tilde{n}^{\mu}
$$

while $(12)$ can be written as

$$
\tilde{n}^{0}\left(x^{\prime}\right)\left[\phi(x), \partial_{0}^{\prime} \phi\left(x^{\prime}\right)\right]_{\Sigma}=i \delta^{3}\left(\mathbf{x}-\mathbf{x}^{\prime}\right) .
$$

This can also be written in the canonical form as

$$
\left[\phi(x), \tilde{\pi}\left(x^{\prime}\right)\right]_{\Sigma}=i \delta^{3}\left(\mathbf{x}-\mathbf{x}^{\prime}\right)
$$

where

$$
\tilde{\pi}=\left|g^{(3)}\right|^{1 / 2} \pi
$$

is not a scalar.

\subsection{A choice of representation}

Let us choose a particular complete orthonormal set of solutions $\left\{f_{k}(x)\right\}$ of Eq. (6). They satisfy

$$
\begin{gathered}
\left(f_{k}, f_{k^{\prime}}\right)=-\left(f_{k}^{*}, f_{k^{\prime}}^{*}\right)=\delta_{k k^{\prime}} \\
\left(f_{k}^{*}, f_{k^{\prime}}\right)=\left(f_{k}, f_{k^{\prime}}^{*}\right)=0 .
\end{gathered}
$$

The field $\phi$ can be expanded as

$$
\phi(x)=\sum_{k} a_{k} f_{k}(x)+a_{k}^{\dagger} f_{k}^{*}(x) .
$$

From (20) and (21) we find

$$
a_{k}=\left(f_{k}, \phi\right), \quad a_{k}^{\dagger}=-\left(f_{k}^{*}, \phi\right),
$$

while from (20) and the canonical commutation relations it follows that

$$
\begin{gathered}
{\left[a_{k}, a_{k^{\prime}}^{\dagger}\right]=\delta_{k k^{\prime}},} \\
{\left[a_{k}, a_{k^{\prime}}\right]=\left[a_{k}^{\dagger}, a_{k^{\prime}}^{\dagger}\right]=0}
\end{gathered}
$$

Therefore, we can interpret $a_{k}$ and $a_{k}^{\dagger}$ as lowering and raising operators, respectively. They induce the representation of the field algebra in the usual way. The vacuum $|0\rangle$ of the corresponding Hilbert space is defined by

$$
a_{k}|0\rangle=0
$$

The operator of the total number of particles is

$$
N=\sum_{k} a_{k}^{\dagger} a_{k}
$$

We also introduce the function $W\left(x, x^{\prime}\right)$, here defined as

$$
W\left(x, x^{\prime}\right)=\sum_{k} f_{k}(x) f_{k}^{*}\left(x^{\prime}\right)
$$


(Later we also study different definitions of $W\left(x, x^{\prime}\right)$.) Using (21), we find that it is a Wightman function

$$
W\left(x, x^{\prime}\right)=\left\langle 0\left|\phi(x) \phi\left(x^{\prime}\right)\right| 0\right\rangle .
$$

From (26) it follows that

$$
W^{*}\left(x, x^{\prime}\right)=W\left(x^{\prime}, x\right) .
$$

From the fact that $f_{k}$ and $f_{k}^{*}$ satisfy Eq. (6) we find

$$
\begin{gathered}
\left(\nabla^{\mu} \partial_{\mu}+m^{2}+\xi R(x)\right) W\left(x, x^{\prime}\right)=0, \\
\left(\nabla^{\prime \mu} \partial_{\mu}^{\prime}+m^{2}+\xi R\left(x^{\prime}\right)\right) W\left(x, x^{\prime}\right)=0 .
\end{gathered}
$$

From (27) and the canonical commutation relations we find that $f_{k}$ and $f_{k}^{*}$ are functions such that

$$
\begin{gathered}
\left.W\left(x, x^{\prime}\right)\right|_{\Sigma}=\left.W\left(x^{\prime}, x\right)\right|_{\Sigma} \\
\left.\partial_{0} \partial_{0}^{\prime} W\left(x, x^{\prime}\right)\right|_{\Sigma}=\left.\partial_{0} \partial_{0}^{\prime} W\left(x^{\prime}, x\right)\right|_{\Sigma} \\
\tilde{n}^{0} \partial_{0}^{\prime}\left[W\left(x, x^{\prime}\right)-W\left(x^{\prime}, x\right)\right]_{\Sigma}=i \delta^{3}\left(\mathbf{x}-\mathbf{x}^{\prime}\right) .
\end{gathered}
$$

\subsection{The current of particle density}

The number operator given by (25) is a global quantity. However, as originally found in [14], a new way of looking into the concept of particles emerges when (22) is put into (25), and (9) and (26) are used. This leads to

$$
N=\int_{\Sigma} d \Sigma^{\mu} \int_{\Sigma} d \Sigma^{\prime \nu} W\left(x, x^{\prime}\right) \stackrel{\leftrightarrow}{\partial_{\mu}} \stackrel{\leftrightarrow}{\partial_{\nu}^{\prime}} \phi(x) \phi\left(x^{\prime}\right)
$$

By interchanging the names of the coordinates $x$ and $x^{\prime}$ and the names of the indices $\mu$ and $\nu$, this can be written as a sum of two equal terms

$$
\begin{aligned}
N & =\frac{1}{2} \int_{\Sigma} d \Sigma^{\mu} \int_{\Sigma} d \Sigma^{\prime \nu} W\left(x, x^{\prime}\right) \stackrel{\leftrightarrow}{\partial_{\mu}} \stackrel{\leftrightarrow}{\partial_{\nu}^{\prime}} \phi(x) \phi\left(x^{\prime}\right) \\
& +\frac{1}{2} \int_{\Sigma} d \Sigma^{\mu} \int_{\Sigma} d \Sigma^{\prime \nu} W\left(x^{\prime}, x\right) \overleftrightarrow{\partial_{\mu}} \stackrel{\leftrightarrow}{\partial_{\nu}^{\prime}} \phi\left(x^{\prime}\right) \phi(x)
\end{aligned}
$$

Using also (28), we see that (35) can be written in the final form as

$$
N=\int_{\Sigma} d \Sigma^{\mu} j_{\mu}(x)
$$

where the vector $j_{\mu}(x)$ is the hermitian operator

$$
j_{\mu}(x)=\int_{\Sigma} d \Sigma^{\prime \nu} \frac{1}{2}\left\{W\left(x, x^{\prime}\right) \overleftrightarrow{\partial_{\mu}} \stackrel{\leftrightarrow}{\partial_{\nu}^{\prime}} \phi(x) \phi\left(x^{\prime}\right)+\text { h.c. }\right\}
$$

Obviously, the vector $j_{\mu}(x)$ should be interpreted as the local current of particle density. The representation of $N$ by (36) and (37) has three advantages with respect to the conventional representation (25). First, it avoids the use of the operators $a_{k}$ and $a_{k}^{\dagger}$ related to a particular choice of the modes $f_{k}(x)$. Second, it is manifestly covariant. Third, the local current $j_{\mu}(x)$ allows to view the concept of particles in a local manner, which the conventional representation does not allow. 
If we put (21) and (26) in (37) and then use (9), (20), (23), and the antisymmetry of the operator $\stackrel{\leftrightarrow}{\partial}_{\mu}$, we find

$$
j_{\mu}=i \sum_{k, k^{\prime}} f_{k}^{*} \stackrel{\leftrightarrow}{\partial_{\mu}} f_{k^{\prime}} a_{k}^{\dagger} a_{k^{\prime}}
$$

From this form we see that $j_{\mu}$ is automatically normally ordered and has the property

$$
j_{\mu}|0\rangle=0
$$

This is actually not surprising because we have started from the operator (25) which is also normally ordered.

Using (6) and (29), we find that the current (37) possesses the property

$$
\nabla^{\mu} j_{\mu}(x)=0 .
$$

This covariant conservation law means that the background gravitational field does not produce particles, provided that a unique (or a preferred) vacuum defined by (24) exists. (This local covariant conservation implies also the global conservation because it provides that (36) does not depend on time. The extra terms in (40) that originate from the fact that $\nabla_{\mu} \neq \partial_{\mu}$ are compensated by the extra terms in (36) that originate from the fact that $d \Sigma^{\mu} \neq\left(d^{3} x, 0,0,0\right)$.) The choice of the vacuum is related to the choice of the function $W\left(x, x^{\prime}\right)$ (see (26) and (27)).

Note that although $j_{\mu}(x)$ is a local operator, some non-local features of the particle concept still remain, because (37) involves an integration over $\Sigma$ on which $x$ lies. Since $\phi\left(x^{\prime}\right)$ satisfies (6) and $W\left(x, x^{\prime}\right)$ satisfies (30), this integral does not depend on $\Sigma$. However, it does depend on the choice of $W\left(x, x^{\prime}\right)$. Note also that the separation between $x$ and $x^{\prime}$ in (37) is spacelike, which softens the non-local features because $W\left(x, x^{\prime}\right)$ decreases rapidly with spacelike separation. As can be explicitly seen with the usual plane-wave modes in Minkowski spacetime (see Sec. 3.4), $W\left(x, x^{\prime}\right)$ is negligible when the spacelike separation is much larger than the Compton wavelength $m^{-1}$.

\subsection{The case of Minkowski spacetime}

In Minkowski spacetime with the metric $g_{\mu \nu}=\operatorname{diag}(1,-1,-1,-1)$, it is most natural to choose the modes $f_{k}$ as the usual plane-wave modes $f_{\mathbf{k}}$, where $\mathbf{k}$ is the 3 -momentum. Using the discrete notation for the summation over $\mathbf{k}$, these modes are

$$
f_{\mathbf{k}}(x)=\frac{e^{-i k x}}{\sqrt{V 2 \omega_{\mathbf{k}}}},
$$

where $\omega_{\mathbf{k}}=\sqrt{\mathbf{k}^{2}+m^{2}}, V$ is the 3 -volume and $k^{\mu}=\left(\omega_{\mathbf{k}}, \mathbf{k}\right)$. Eq. (38) becomes

$$
j_{\mu}=\frac{1}{V} \sum_{\mathbf{k}, \mathbf{k}^{\prime}} \frac{k_{\mu}+k_{\mu}^{\prime}}{2 \sqrt{\omega_{\mathbf{k}} \omega_{\mathbf{k}^{\prime}}}} e^{i\left(k-k^{\prime}\right) x} a_{\mathbf{k}}^{\dagger} a_{\mathbf{k}^{\prime}}
$$

The physical content is more obvious from the space-integrated operator

$$
\tilde{J}_{\mu} \equiv \int d^{3} x j_{\mu}(x)=\sum_{\mathbf{k}} \frac{k_{\mu}}{\omega_{\mathbf{k}}} a_{\mathbf{k}}^{\dagger} a_{\mathbf{k}},
$$

where the tilde on $\tilde{J}_{\mu}$ denotes that it does not transform as a 4 -vector. The quantity $k^{i} / \omega_{\mathbf{k}}$ is the 3 -velocity $v^{i}$, so we find

$$
\tilde{\mathbf{J}}\left|n_{q}\right\rangle=\mathbf{v} n_{q}\left|n_{q}\right\rangle
$$


where $\left|n_{q}\right\rangle=\left(n_{q} !\right)^{-1 / 2}\left(a_{q}^{\dagger}\right)^{n_{q}}|0\rangle$ is the state with $n_{q}$ particles with the momentum $q$. Eqs. (43) and (44) support the interpretation of $j_{\mu}$ as the particle current.

The two-point function (26) is

$$
W\left(x, x^{\prime}\right)=\frac{1}{V} \sum_{\mathbf{k}} \frac{e^{-i k\left(x-x^{\prime}\right)}}{2 \omega_{\mathbf{k}}} .
$$

In the infinite-volume limit, we perform the replacement

$$
\sum_{\mathbf{k}} \rightarrow V \int \frac{d^{3} k}{(2 \pi)^{3}}
$$

so (45) takes the usual form

$$
W\left(x, x^{\prime}\right)=\int \frac{d^{3} k}{(2 \pi)^{3} 2 \omega_{\mathbf{k}}} e^{-i k\left(x-x^{\prime}\right)} .
$$

The properties of this function, often denoted by $\Delta\left(x-x^{\prime}\right)$ [19], are well known. In particular, for $t=t^{\prime}$ and $\left|\mathbf{x}-\mathbf{x}^{\prime}\right| \equiv r$, it behaves asymptotically as

$$
\Delta(r) \propto \frac{\sqrt{m r}}{r^{2}} e^{-m r} \text { for } r m \gg 1 .
$$

For $m=0$, the function $W\left(x, x^{\prime}\right)$ is

$$
\left.\Delta\left(x-x^{\prime}\right)\right|_{m=0}=-\frac{1}{4 \pi^{2}}\left[\frac{1}{\left(x-x^{\prime}\right)^{2}}+i \pi \delta\left(\left(x-x^{\prime}\right)^{2}\right)\right] .
$$

The function (47) is also equal to $G^{+}\left(x, x^{\prime}\right)$, where $i G^{+}\left(x, x^{\prime}\right)$ is equal to the Green function

$$
G\left(x, x^{\prime}\right)=\int \frac{d^{4} k}{(2 \pi)^{4}} \frac{e^{-i k\left(x-x^{\prime}\right)}}{k^{2}-m^{2}},
$$

calculated with the appropriately chosen contour of integration over $k_{0}$ [2].

\subsection{The question of positivity of the local number of particles}

Eq. (36) can be written as

$$
N=\int_{\Sigma} d^{3} x\left|g^{(3)}\right|^{1 / 2} n(x)
$$

where

$$
n=n^{\mu} j_{\mu}
$$

Since the total number of particles (25) is a non-negative operator (in the sense that the expected value $\langle\psi|N| \psi\rangle$ is non-negative for any state $|\psi\rangle$ ), one could expect that the local density of particles $n(x)$ is also a non-negative operator. For example, in the Minkowski case, for $|q\rangle \equiv$ $a_{\mathbf{q}}^{\dagger}|0\rangle$, we find $\langle q|n| q\rangle=\left\langle q\left|j_{0}\right| q\right\rangle=V^{-1}$, which is a positive constant. However, for $|\psi\rangle=$ $2^{-1 / 2}\left(\left|q_{1}\right\rangle+\left|q_{2}\right\rangle\right)$, where $q_{1} \neq q_{2}$, we find

$$
\left\langle\psi\left|j_{0}\right| \psi\right\rangle=\frac{1}{V}\left[1+\frac{\omega_{\mathbf{q}_{1}}+\omega_{\mathbf{q}_{2}}}{2 \sqrt{\omega_{\mathbf{q}_{1}} \omega_{\mathbf{q}_{2}}}} \cos \left(q_{1}-q_{2}\right) x\right],
$$

which is negative for some values of $x$, provided that $\omega_{\mathbf{q}_{1}} \neq \omega_{\mathbf{q}_{2}}$. Therefore, the particle density $n(x)$ is not a non-negative operator. Is this in contradiction with the experimental fact that the 
number of particles in a small volume $\sigma \subset \Sigma$ cannot be negative? The answer is no! When the position of a particle is measured with some accuracy such that it is (almost) certain that the particle is localized inside $\sigma$, then the state $|\psi\rangle$ of the particle is a localized state, such that the corresponding wave function vanishes (or is negligible) outside of $\sigma$. Consequently, the quantity $\langle\psi|n(x)| \psi\rangle$ vanishes (or is negligible) outside of $\sigma$. Therefore, the total number of particles in this state is (approximately) equal to

$$
\mathcal{N}_{\sigma}=\int_{\sigma} d^{3} x\left|g^{(3)}\right|^{1 / 2}\langle\psi|n(x)| \psi\rangle .
$$

Since the total number of particles cannot be negative, it follows that the number of particles inside $\sigma$ is non-negative for such a localized state.

Let us illustrate the heuristic arguments above by a concrete example. We study the case of Minkowski spacetime with infinite volume $V$, so the field expansion (21) becomes

$$
\phi(x)=\int d^{3} k\left[a_{\mathbf{k}} f_{\mathbf{k}}(x)+a_{\mathbf{k}}^{\dagger} f_{\mathbf{k}}^{*}(x)\right]
$$

where

$$
f_{\mathbf{k}}(x)=\frac{e^{-i k x}}{\sqrt{(2 \pi)^{3} 2 \omega_{\mathbf{k}}}} .
$$

Let the state of the system be the one-particle state

$$
|\mathbf{y}\rangle \equiv c^{-1 / 2} \phi(0, \mathbf{y})|0\rangle \text {. }
$$

The constant $c$ is the norm determined by the requirement that $\langle\mathbf{y} \mid \mathbf{y}\rangle=1$, so it is equal to

$$
c=\Delta^{+}(0)=\Delta^{-}(0),
$$

where

$$
\Delta^{ \pm}(x)=\int \frac{d^{3} k}{(2 \pi)^{3} 2 \omega_{\mathbf{k}}} e^{\mp i k x}
$$

The wave function corresponding to the state (57) is

$$
\psi_{\mathbf{y}}(x)=\tilde{c}\langle 0|\phi(x)| \mathbf{y}\rangle=\tilde{c} c^{-1 / 2} \Delta^{+}(t, \mathbf{x}-\mathbf{y}),
$$

where $\tilde{c}$ is a constant chosen such that the wave function is normalized in an appropriate way. This wave function is related to a Lorentz-invariant notion of particle detection [20]. Using the fact that $W\left(x, x^{\prime}\right)=\Delta^{+}\left(x-x^{\prime}\right)$, a straightforward calculation gives

$$
\left\langle\mathbf{y}\left|j_{0}(x)\right| \mathbf{y}\right\rangle=\frac{1}{2}\left[\frac{\Delta^{+}(t, \mathbf{x}-\mathbf{y}) \delta^{-}(t, \mathbf{x}-\mathbf{y})}{\Delta^{+}(0)}+\text { c.c. }\right],
$$

where

$$
\delta^{ \pm}(x)=\int \frac{d^{3} k}{(2 \pi)^{3}} e^{\mp i k x}
$$

In particular, at $t=0$

$$
\left\langle\mathbf{y}\left|j_{0}(0, \mathbf{x})\right| \mathbf{y}\right\rangle=\delta^{3}(\mathbf{x}-\mathbf{y}),
$$

which implies that the state (57) has a non-negative and strictly localized particle density at $t=0$. Note that the wave function (60) is not strictly localized at $t=0$, but decreases rapidly with $|\mathbf{x}-\mathbf{y}|$ (see (48) and (49)). 
Note also that in a typical physical situation in which particles are detected (see Sec. 2), the state $|\psi\rangle$ corresponds to $N$ particles, where each particle is localized inside one of $N$ lumps. In such a situation, (54) represents the number of particles in one of the lumps, so this number is equal to one.

We also stress that negative particle densities do not appear in non-relativistic quantum field theory (see Appendix A). Heuristically, this can be seen from the frequency-dependent factor that multiplies the cosine function in (53). Since in the non-relativistic limit $\omega_{q} \simeq m$, this factor becomes equal to 1, leading to a non-negative density.

Note finally that, in general, the particle density (that may be negative) is not the same thing as the probability density (that cannot be negative) of finding the particle at a position $\mathbf{x}$. The probability density in a state $|\psi\rangle$ is equal to $|\langle\tilde{\mathbf{x}} \mid \psi\rangle|^{2}$, where $|\tilde{\mathbf{x}}\rangle$ is an eigenstate of the position operator $\mathbf{X}$ with the eigenvalue $\mathbf{x}$. For the relativistic case, the problem of finding the operator $\mathbf{X}$ and the states $|\tilde{\mathbf{x}}\rangle$ is not yet satisfactorily solved. In the nonrelativistic limit, the states $|\tilde{\mathbf{x}}\rangle$ are equal to the states $|\mathbf{x}\rangle$ defined by (57).

\subsection{Particle production by non-gravitational interactions}

Let us now study the case in which a non-gravitational interaction represented by $\mathcal{L}_{\text {int }}=-\mathcal{H}_{\text {int }}$ is also present. In this case, the equation of motion is

$$
\left(\nabla^{\mu} \partial_{\mu}+m^{2}+\xi R\right) \phi=J
$$

where $J(x)$ is a local operator containing $\phi$ and/or other dynamical quantum fields. Since it describes the interaction, it does not contain terms linear in quantum fields. (For example, when $\mathcal{L}_{\text {int }}=-\lambda \phi^{4} / 4$, then $\left.J(x)=-\lambda \phi^{3}(x)\right)$. We propose that even in this general case the particle current is given by (37)

$$
j_{\mu}(x)=\int_{\Sigma} d \Sigma^{\prime \nu} \frac{1}{2}\left\{W\left(x, x^{\prime}\right) \stackrel{\leftrightarrow}{\partial_{\mu}} \stackrel{\leftrightarrow}{\partial_{\nu}^{\prime}} \phi(x) \phi\left(x^{\prime}\right)+\text { h.c. }\right\},
$$

where $W\left(x, x^{\prime}\right)$ is the same function as before, satisfying the "free" equations

$$
\begin{aligned}
& \left(\nabla^{\mu} \partial_{\mu}+m^{2}+\xi R(x)\right) W\left(x, x^{\prime}\right)=0 \\
& \left(\nabla^{\prime \mu} \partial_{\mu}^{\prime}+m^{2}+\xi R\left(x^{\prime}\right)\right) W\left(x, x^{\prime}\right)=0,
\end{aligned}
$$

and having the expansion

$$
W\left(x, x^{\prime}\right)=\sum_{k} f_{k}(x) f_{k}^{*}\left(x^{\prime}\right) .
$$

As we show below, such an ansatz leads to particle production consistent with the conventional approach to particle production caused by a non-gravitational interaction $\mathcal{L}_{\text {int }}$.

The field $\phi$ in (65) satisfies (64). Therefore, using (66), we find

$$
\nabla^{\mu} j_{\mu}(x)=\int_{\Sigma} d \Sigma^{\prime \nu} \frac{1}{2}\left\{W\left(x, x^{\prime}\right) \overleftrightarrow{\partial_{\nu}^{\prime}} J(x) \phi\left(x^{\prime}\right)+\text { h.c. }\right\}
$$

Note that only $x$ (not $x^{\prime}$ ) appears as the argument of $J$ on the right-hand side of (69), implying that $J$ plays a strictly local role in particle production.

Let us now show that our covariant description (69) of particle production is consistent with the conventional approach to particle production caused by a non-gravitational interaction. Let $\Sigma(t)$ denote some foliation of spacetime into Cauchy spacelike hypersurfaces. The total mean number of particles at the time $t$ in a state $|\psi\rangle$ is

$$
\mathcal{N}(t)=\langle\psi|N(t)| \psi\rangle,
$$


where

$$
N(t)=\int_{\Sigma(t)} d \Sigma^{\mu} j_{\mu}(t, \mathbf{x})
$$

Equation (70) is written in the Heisenberg picture. However, matrix elements do not depend on picture. We introduce the interaction picture, where the interaction Hamiltonian is the part of the Hamiltonian that generates the right-hand side of (64). The state $|\psi\rangle$ transforms to a time-dependent state

$$
\left|\psi_{\text {int }}(t)\right\rangle=U_{\text {int }}(t)|\psi\rangle .
$$

Here the unitary operator $U_{\text {int }}(t)$ satisfies

$$
i \frac{d}{d t} U_{\mathrm{int}}(t)=\tilde{H}_{\mathrm{int}}(t) U_{\mathrm{int}}(t),
$$

where

$$
\tilde{H}_{\text {int }}(t)=\int_{\Sigma(t)} d^{3} x|g|^{1 / 2} \mathcal{H}_{\text {int }}(x) .
$$

Note that Eqs. (73) and (74) are not manifestly covariant. The covariance of the time-evolution law is discussed in Sec. 3.9. In the interaction picture, the field $\phi$ satisfies the free equation (6), so the expansion (21) can be used. This, together with (71), (65), and (68), implies that $N(t)$ transforms to

$$
N_{\mathrm{int}}=\sum_{k} a_{k}^{\dagger} a_{k}
$$

Therefore, (70) can be written as

$$
\mathcal{N}(t)=\left\langle\psi_{\text {int }}(t)\left|N_{\text {int }}\right| \psi_{\text {int }}(t)\right\rangle=\sum_{k}\left\langle\psi_{\text {int }}(t)\left|a_{k}^{\dagger} a_{k}\right| \psi_{\text {int }}(t)\right\rangle,
$$

which is the usual formula that describes particle production caused by a quantum non-gravitational interaction. This is most easily seen when $t \rightarrow \infty$ and the solution of (73) is chosen such that $\left|\psi_{\text {int }}(t \rightarrow-\infty)\right\rangle=|\psi\rangle$, in which case $U_{\text {int }}$ is the $S$-matrix operator.

\subsection{Particle production by the gravitational background}

So far, our discussion has been based on a particular choice of the modes $\left\{f_{k}(x)\right\}$ as preferred modes. In practice, they are chosen such that $f_{k}$ are positive frequency solutions of (6). However, when metric is time dependent, then such preferred modes do not exist. In the conventional approach to the concept of particles, this is related to particle production by the gravitational background [2, 6]. In this case, one introduces a new set of functions $u_{l}(x)$ for each time $t$, such that $u_{l}(x)$ are positive-frequency modes at that time. This means that the modes $u_{l}$ possess an extra time dependence, i.e., they become functions of the form $u_{l}(x ; t)$. These functions do not satisfy (6). However, the functions $u_{l}(x ; \tau)$ satisfy (6), provided that $\tau$ is kept fixed when the derivative $\partial_{\mu}$ acts on $u_{l}$. To describe the local particle production, we take

$$
W\left(x, x^{\prime}\right)=\sum_{l} u_{l}(x ; t) u_{l}^{*}\left(x^{\prime} ; t^{\prime}\right)
$$

instead of (26). Since $u_{l}(x ; t)$ do not satisfy (6), the function (77) does not satisfy (29). Instead, we have

$$
\left(\nabla^{\mu} \partial_{\mu}+m^{2}+\xi R(x)\right) W\left(x, x^{\prime}\right) \equiv-K\left(x, x^{\prime}\right) \neq 0 .
$$


Using (6) and (78) in (37), we find a relation similar to (69):

$$
\nabla^{\mu} j_{\mu}(x)=\int_{\Sigma} d \Sigma^{\prime \nu} \frac{1}{2}\left\{K\left(x, x^{\prime}\right) \overleftrightarrow{\partial_{\nu}^{\prime}} \phi(x) \phi\left(x^{\prime}\right)+\text { h.c. }\right\}
$$

This local description of particle production is consistent with the usual global description based on the Bogoliubov transformation. This is because (71) and (37) with (77) and (21) lead to

$$
N(t)=\sum_{l} A_{l}^{\dagger}(t) A_{l}(t)
$$

where

$$
\begin{gathered}
A_{l}(t)=\sum_{k} \alpha_{l k}^{*}(t) a_{k}-\beta_{l k}^{*}(t) a_{k}^{\dagger}, \\
\alpha_{l k}(t)=\left(f_{k}, u_{l}\right), \quad \beta_{l k}(t)=-\left(f_{k}^{*}, u_{l}\right) .
\end{gathered}
$$

The time dependence of the Bogoliubov coefficients $\alpha_{l k}(t)$ and $\beta_{l k}(t)$ is related to the extra time dependence of the modes $u_{l}(x ; t)$. If we assume that the change of the average number of particles is slow, i.e., that

$$
\partial_{t} A_{l}(t) \approx 0
$$

which occurs when

$$
\left.\partial_{t} u_{l}(\tau, \mathbf{x} ; t)\right|_{\tau=t} \approx 0,
$$

then the Bogoliubov coefficients (82) are equal to the usual Bogoliubov coefficients. This approximation is nothing else but the adiabatic approximation, which is a usual part of the convential description of particle production [6].

\subsection{The natural choice of $W\left(x, x^{\prime}\right)$}

In general, there is no universal natural choice for the modes $u_{l}(x ; t)$. In particular, in a given spacetime, the choice of the natural modes $u_{l}(x ; t)$ may depend on the observer. If different observers (that use different coordinates) use different modes for the choice of (77), then the coordinate transformation alone does not describe how the particle current is seen by different observers. In this sense, the particle current is not really covariant. There are also other problems related to the case in which different observers use different modes [21].

Since covariance was our original aim, it is desirable to find a universal natural choice of $W\left(x, x^{\prime}\right)$, such that, in Minkowski spacetime, it reduces to the usual plane-wave expansion (47). Such a choice exists. This is based on the fact that the Feynman Green function $G_{F}\left(x, x^{\prime}\right)$ can be calculated using the Schwinger-DeWitt method and that the knowledge of the Feynman Green function automatically yields the knowledge of other Green functions [2, 16], such as $G^{+}\left(x, x^{\prime}\right)$. The Schwinger-DeWitt method does not require a choice of a particular set of modes. Therefore, we propose

$$
W\left(x, x^{\prime}\right)=G^{+}\left(x, x^{\prime}\right)
$$

where $G^{+}$is calculated from $G_{F}$ given by

$$
G_{F}\left(x, x^{\prime}\right)=i\left[g(x) g\left(x^{\prime}\right)\right]^{-1 / 4} \int_{0}^{\infty} d s\left\langle x, s \mid x^{\prime}, 0\right\rangle .
$$

The function $\left\langle x, s \mid x^{\prime}, 0\right\rangle$ is determined by the function $\sigma\left(x, x^{\prime}\right)$ and its derivatives, where $\sigma\left(x, x^{\prime}\right)$ is one half of the square of the geodesic distance between $x$ and $x^{\prime}[16]$. Therefore, the Green function $G_{F}$ is unique, provided that a geodesic connecting $x$ and $x^{\prime}$ is chosen. When $x$ and $x^{\prime}$ are sufficiently close to one another, then there is only one such geodesic. In this case, the 
adiabatic expansion [2, 16] of $G_{F}$ can be used. If Riemann normal coordinates are spanned around $x^{\prime}$, then all Green functions can be written as [2, 22]

$$
G\left(x, x^{\prime}\right)=|g(x)|^{-1 / 4} \int \frac{d^{4} k}{(2 \pi)^{4}} e^{-i k\left(x-x^{\prime}\right)} \mathcal{G}\left(k ; x^{\prime}\right),
$$

where

$$
\mathcal{G}\left(k ; x^{\prime}\right)=\sum_{n=0}^{\infty} \mathcal{G}_{n}\left(k ; x^{\prime}\right)
$$

and $\mathcal{G}_{n}\left(k ; x^{\prime}\right)$ have $n$ derivatives of the metric at $x^{\prime}$. The two lowest functions of the adiabatic expansion (88) are the same as in Minkowski spacetime, i.e., $\mathcal{G}_{0}=\left(k^{2}-m^{2}\right)^{-1}, \mathcal{G}_{1}=0$. Depending on the choice of the integration contour over $k_{0}$, from (87) one can calculate $G_{F}, G^{+}$, or any other Green function. In particular, $G^{+}$satisfies

$$
\begin{gathered}
\left(\nabla^{\mu} \partial_{\mu}+m^{2}+\xi R(x)\right) G^{+}\left(x, x^{\prime}\right)=0, \\
\left(\nabla^{\prime \mu} \partial_{\mu}^{\prime}+m^{2}+\xi R\left(x^{\prime}\right)\right) G^{+}\left(x, x^{\prime}\right)=0 .
\end{gathered}
$$

Since $G^{+}\left(x, x^{\prime}\right)$ decreases rapidly with spacelike separation, the contributions to (37) from large spacelike separations may be negligible. Therefore, for practical calculations, it may be sufficient to calculate $G^{+}\left(x, x^{\prime}\right)$ only for small spacelike separations, in which case the first few terms of the adiabatic expansion are sufficient.

When $x$ and $x^{\prime}$ are not close to one another, then there may be more than one geodesic connecting $x$ and $x^{\prime}$. In this case, $\left\langle x, s \mid x^{\prime}, 0\right\rangle$ depends on the choice of the geodesic, the point $x$ is not contained in the subspace covered by the Riemann normal coordinates spanned around $x^{\prime}$ and the adiabatic expansion does not work. Nevertheless, $\left\langle x, s \mid x^{\prime}, 0\right\rangle$ can be computed for any choice of the geodesic connecting $x$ and $x^{\prime}$. The resulting Green function $G^{+}$satisfies (89) and (90). To define the exact unique particle current, we need a natural generalization of $G^{+}$to the case with more than one geodesic connecting $x$ and $x^{\prime}$. The most natural choice is the 2-point function $\bar{G}^{+}$defined as the average over all geodesics connecting $x$ and $x^{\prime}$. Assuming that there are $N$ such geodesics, this average is (for any Green function $G$ )

$$
\bar{G}\left(x, x^{\prime}\right)=N^{-1} \sum_{a=1}^{N} G\left(x, x^{\prime} ; \sigma_{a}\right),
$$

where $G\left(x, x^{\prime} ; \sigma_{a}\right)$ is the Green function $G\left(x, x^{\prime}\right)$ calculated with respect to the geodesic $\sigma_{a}$.

In the case with a continuous set of geodesics connecting $x$ and $x^{\prime}$, the generalization of (91) is more complicated. At the point $x^{\prime}$ we span the flat Minkowski tangent space. Then we perform the Wick rotation on the tangent space which transforms it to a Euclidean tangent space. Each geodesic emanating from $x^{\prime}$ is uniquely determined by the space angle $\Omega=\left(\varphi, \vartheta_{1}, \vartheta_{2}\right)$ in the Euclidean tangent space because the space angle determines the direction of a geodesic emanating from $x^{\prime}$. Therefore, the measure on the set of all geodesics emanating from $x^{\prime}$ is (see, for example, 23])

$$
\int d \Omega_{x^{\prime}}=\int_{0}^{2 \pi} d \varphi \int_{0}^{\pi} \sin \vartheta_{1} d \vartheta_{1} \int_{0}^{\pi} \sin ^{2} \vartheta_{2} d \vartheta_{2}
$$

where geodesics emanating in two opposite directions are counted as two different geodesics. Of course, not all geodesics emanating from $x^{\prime}$ cross the point $x$, but some of them do. The Green function corresponding to a geodesic determined by its space angle $\Omega_{x^{\prime}}$ at $x^{\prime}$ and crossing the 
point $x$ is denoted by $G\left(x, x^{\prime} ; \Omega_{x^{\prime}}\right)$. Therefore, we introduce the averaged Green function

$$
G_{x^{\prime}}\left(x, x^{\prime}\right)=\frac{\int^{\prime} d \Omega_{x^{\prime}} G\left(x, x^{\prime} ; \Omega_{x^{\prime}}\right)}{\int^{\prime} d \Omega_{x^{\prime}}},
$$

where $\int^{\prime}$ denotes integration only over those space angles for which the corresponding geodesic crosses the point $x$. However, a geodesic connecting $x$ and $x^{\prime}$ can also be specified by its angle in the Euclidean tangent space spanned at $x$, resulting in the averaged Green function

$$
G_{x}\left(x, x^{\prime}\right)=\frac{\int^{\prime} d \Omega_{x} G\left(x, x^{\prime} ; \Omega_{x}\right)}{\int^{\prime} d \Omega_{x}} .
$$

In general, (93) and (94) are not equal. To treat the points $x$ and $x^{\prime}$ in a symmetric way, we introduce the symmetrized Green function

$$
\bar{G}\left(x, x^{\prime}\right)=\frac{1}{2}\left[G_{x}\left(x, x^{\prime}\right)+G_{x^{\prime}}\left(x, x^{\prime}\right)\right]
$$

The symmetrized Green function satisfies (28), i.e.

$$
\bar{G}^{*}\left(x, x^{\prime}\right)=\bar{G}\left(x^{\prime}, x\right),
$$

provided that $G$ is a Green function such that $G_{x}^{*}\left(x, x^{\prime}\right)=G_{x}\left(x^{\prime}, x\right)$ and $G_{x^{\prime}}^{*}\left(x, x^{\prime}\right)=G_{x^{\prime}}\left(x^{\prime}, x\right)$.

Now the most natural choice for the particle current in arbitrary curved background is

$$
j_{\mu}(x)=\int_{\Sigma} d \Sigma^{\prime \nu} \frac{1}{2}\left\{\bar{G}^{+}\left(x, x^{\prime}\right) \stackrel{\leftrightarrow}{\partial_{\mu}} \stackrel{\leftrightarrow}{\partial_{\nu}^{\prime}} \phi(x) \phi\left(x^{\prime}\right)+\text { h.c. }\right\}
$$

Since the function $\bar{G}^{+}\left(x, x^{\prime}\right)$ satisfies (89) and (90), it follows that the particle current (97) does not depend on the choice of $\Sigma$ and is conserved

$$
\nabla^{\mu} j_{\mu}(x)=0
$$

provided that the field satisfies (6). Note that the corresponding definition of particles does not always correspond to the quantities detected by "particle detectors" of the Unruh-DeWitt type [4, 5]. Instead, the number of particles resulting from (97) is determined by a well-defined hermitian operator that does not require a model of a particle detector, just as is the case for all other observables in quantum mechanics. Moreover, since the observable $\phi(x)$ in (97) does not require a choice of representation of field algebra, the definition of particles based on $\bar{G}^{+}$ does not require the choice of representation either. This allows us to treat the particles in the framework of algebraic quantum field theory in curved background [24]. Furthermore, as noted by Unruh 狛, only one definition of particles can correspond to the real world, in the sense that their stress-energy contributes to the gravitational field. Since the definition of particles based on $\bar{G}^{+}$is universal, unique, and really covariant, it might be that these are the particles that correspond to the real world. Similarly, from the discussion in Sec. 2, it seems that these particles might correspond to the objects detected by real detectors (such as a Wilson chamber or a Geiger-Müller counter) in real experiments. If such an interpretation of these particles is correct, then classical gravitational backgrounds do not produce real particles, in agreement with some other results [13, 25, 26, 27, 28]. Note that this does not necessarily imply that 
black holes do not radiate. It is possible that quantum gravity provides a correct mechanism for black hole radiation. Whatever the mechanism of the particle creation near the horizon might be, one should expect an approximately thermal distribution of escaped particles as seen by a distant observer. The thermal distribution is a consequence of the exponential red shift and can be understood even by classical physics [29, 30], without any assumption on the physical mechanism that causes particle creation near the horizon.

\subsection{Covariance of the time evolution}

As we have seen, when particle production occurs, then the function $W\left(x, x^{\prime}\right)$ or the field $\phi\left(x^{\prime}\right)$ does not satisfy the "free" equation of motion (30) or (6), respectively. Consequently, the current $j_{\mu}(x)$ depends not only on the point $x$, but also on the choice of $\Sigma$ on which $x$ lies.

This suggests that there exists a preferred foliation of spacetime. Such a result should not be surprising because many other aspects of quantum theory seem to require the existence of a preferred time coordinate. However, it may be surprising that, in our analysis, the need for a preferred time coordinate has emerged from the requirement of general covariance (of the concept od particles). Below we argue that the principle of general covariance (together with some other natural principles) may help us to determine this preferred time coordinate.

The time evolution law (73) written in the Heisenberg picture is

$$
i \frac{d}{d t} U(t)=\tilde{H}(t) U(t)
$$

where $($ see $(74))$

$$
\tilde{H}(t)=\int_{\Sigma(t)} d^{3} x|g|^{1 / 2} \mathcal{H}(x)
$$

$\mathcal{H}=T_{0}^{0}$, and

$$
T_{\nu}^{\mu}=\frac{\partial \mathcal{L}}{\partial\left(\partial_{\mu} \phi\right)} \partial_{\nu} \phi-g_{\nu}^{\mu} \mathcal{L}
$$

Similarly, in the Schrödinger picture

$$
i \frac{d}{d t} \Psi[\phi, t)=\tilde{H} \Psi[\phi, t),
$$

where $\Psi[\phi, t)$ is a functional with respect to $\phi(\mathbf{x})$ and a function with respect to $t$, while the Hamiltonian $\tilde{H}$ is given by (100) in which $\tilde{\pi}(\mathbf{x})=-i \delta / \delta \phi(\mathbf{x})$ (see (18)). This time-evolution law is not manifestly covariant. This is because $t$ and $\tilde{H}$ are not scalars. This law would become covariant if $d t \tilde{H}$ could be replaced with $d \tau H$, where

$$
\begin{gathered}
d \tau=n_{\mu} d x^{\mu}=\frac{g_{0 \mu}}{\sqrt{g_{00}}} d x^{\mu} \\
H=\int_{\Sigma} d \Sigma^{\mu} n^{\nu} T_{\mu \nu}=\int_{\Sigma} d^{3} x\left|g^{(3)}\right|^{1 / 2} \frac{g_{0 \alpha} T_{0}^{\alpha}}{g_{00}}
\end{gathered}
$$

are scalars. It is easy to see that $d t \tilde{H}=d \tau H$ for any $T_{\nu}^{\mu}$ if and only if

$$
g_{0 i}=0 \text { and } \partial_{i} g_{00}=0 .
$$

In this case, $d \tau=\sqrt{g_{00}(t)} d t$, so the metric takes the form

$$
d s^{2}=d \tau^{2}-g_{i j}(\tau, \mathbf{x}) d x^{i} d x^{j}
$$


The metric can always be written in this form. The coordinates for which the metric takes the form (106) are known as Gaussian coordinates [31].

The results above can be reinterpreted in the following way. The covariant time evolution determined by (103) and (104) depends on the foliation of spacetime into Cauchy spacelike hypersurfaces. The choice of foliation corresponds to the choice of coordinates for which $t=$ constant on $\Sigma$. Therefore, we can determine the time evolution uniquelly by giving a preferred status to a set of coordinates. The Gaussian coordinates are special because only in these coordinates the time-evolution law takes the canonical form (99), required by the usual rules of quantum mechanics. This shows that the requirement of covariance of the time-evolution law leads to Gaussian coordinates as preferred coordinates.

However, for a given spacetime, the Gaussian coordinates are not unique. One needs an additional criterion for choosing them. For example, a reasonable additional criterion is the requirement that there should be no coordinate singularity in these coordinates, but this requirement still does not make the Gaussian coordinates unique. An interesting attempt to choose the Gaussian coordinates in a unique way is given in [32, 33]. However, in the approach of [32, 33], the coordinate singularities such as horizons are not avoided. In the case of a Schwarzschild black hole, a particularly interesting choice of the Gaussian coordinates is the Lemaitre coordinates, in which there is no coordinate singularity. In these coordinates, the Schwarzschild black hole is time dependent, the Hawking radiation looks thermal to a distant outside observer, but the absence of the horizon implies a unitary time evolution which resolves the black-hole information paradox [34].

\section{Complex scalar field}

To explore the similarity between the current of particles and that of charge, we generalize some results for hermitian scalar fields to the case of complex scalar fields. We do not discuss those aspects the generalization of which is obvious. We also study the scalar QED, i.e., the interaction of complex scalar fields with classical and quantum electromagnetic fields.

\subsection{Particle and charge currents}

The complex field $\phi$ can be written in terms of hermitian fields $\phi_{1}$ and $\phi_{2}$ as

$$
\phi=\frac{\phi_{1}+i \phi_{2}}{\sqrt{2}}
$$

The independent fields $\phi_{1}$ and $\phi_{2}$ satisfy the same canonical commutation relations as the hermitian scalar field of Sec. 3, with an additional property $\left[\phi_{1}(x), \phi_{2}\left(x^{\prime}\right)\right]=0$. Therefore, the canonical commutation relations can be written as

$$
\begin{gathered}
{\left[\phi^{\dagger}(x), \phi\left(x^{\prime}\right)\right]_{\Sigma}=\left[\partial_{0} \phi^{\dagger}(x), \partial_{0}^{\prime} \phi\left(x^{\prime}\right)\right]_{\Sigma}=0,} \\
\tilde{n}^{0}\left(x^{\prime}\right)\left[\phi^{\dagger}(x), \partial_{0}^{\prime} \phi\left(x^{\prime}\right)\right]_{\Sigma}=i \delta^{3}\left(\mathbf{x}-\mathbf{x}^{\prime}\right) .
\end{gathered}
$$

The complex field $\phi$ satisfies (6) and can be expanded as

$$
\begin{aligned}
\phi(x) & =\sum_{k} a_{k} f_{k}(x)+b_{k}^{\dagger} f_{k}^{*}(x), \\
\phi^{\dagger}(x) & =\sum_{k} a_{k}^{\dagger} f_{k}^{*}(x)+b_{k} f_{k}(x) .
\end{aligned}
$$


The operators $a_{k}\left(a_{k}^{\dagger}\right)$ destroy (create) particles, while $b_{k}\left(b_{k}^{\dagger}\right)$ destroy (create) antiparticles. They satisfy the corresponding algebra of lowering and raising operators and can be expressed as

$$
\begin{gathered}
a_{k}=\left(f_{k}, \phi\right), \quad b_{k}^{\dagger}=-\left(f_{k}^{*}, \phi\right), \\
a_{k}^{\dagger}=-\left(f_{k}^{*}, \phi^{\dagger}\right), \quad b_{k}=\left(f_{k}, \phi^{\dagger}\right) .
\end{gathered}
$$

We introduce two global quantities:

$$
N^{( \pm)}=\sum_{k} a_{k}^{\dagger} a_{k} \pm b_{k}^{\dagger} b_{k}
$$

Here $N^{(+)}$is the total number of particles, while $N^{(-)}$is the total charge. In a way similar to the case of hermitian field, we find the covariant expression

$$
N^{( \pm)}=\int_{\Sigma} d \Sigma^{\mu} j_{\mu}^{( \pm)}(x)
$$

where

$$
j_{\mu}^{( \pm)}(x)=\int_{\Sigma} d \Sigma^{\prime \nu} \frac{1}{2}\left\{W\left(x, x^{\prime}\right) \overleftrightarrow{\partial_{\mu}} \stackrel{\leftrightarrow}{\partial_{\nu}^{\prime}}\left[\phi^{\dagger}(x) \phi\left(x^{\prime}\right) \pm \phi(x) \phi^{\dagger}\left(x^{\prime}\right)\right]+\text { h.c. }\right\}
$$

and $W\left(x, x^{\prime}\right)$ is given by (26). Obviously, $j_{\mu}^{(+)}$should be interpreted as a current of particles, while $j_{\mu}^{(-)}$should be interpreted as a current of charge. These two currents can also be written as

$$
j_{\mu}^{( \pm)}=j_{\mu}^{(P)} \pm j_{\mu}^{(A)}
$$

where

$$
\begin{aligned}
& j_{\mu}^{(P)}(x)=\int_{\Sigma} d \Sigma^{\prime \nu} \frac{1}{2}\left\{W\left(x, x^{\prime}\right) \stackrel{\leftrightarrow}{\partial_{\mu}} \stackrel{\leftrightarrow}{\partial_{\nu}^{\prime}} \phi^{\dagger}(x) \phi\left(x^{\prime}\right)+\text { h.c. }\right\} \\
& j_{\mu}^{(A)}(x)=\int_{\Sigma} d \Sigma^{\prime \nu} \frac{1}{2}\left\{W\left(x, x^{\prime}\right) \overleftrightarrow{\partial_{\mu}} \overleftrightarrow{\partial_{\nu}^{\prime}} \phi(x) \phi^{\dagger}\left(x^{\prime}\right)+\text { h.c. }\right\}
\end{aligned}
$$

are the currents of particles and antiparticles, respectively. It is also instructive to write the currents in terms of hermitian fields $\phi_{1}$ and $\phi_{2}$. The current of particles is a sum of two currents of the form (37);

$$
j_{\mu}^{(+)}(x)=\int_{\Sigma} d \Sigma^{\prime \nu} \frac{1}{2}\left\{W\left(x, x^{\prime}\right) \stackrel{\leftrightarrow}{\partial_{\mu}} \stackrel{\leftrightarrow}{\partial_{\nu}^{\prime}}\left[\phi_{1}(x) \phi_{1}\left(x^{\prime}\right)+\phi_{2}(x) \phi_{2}\left(x^{\prime}\right)\right]+\text { h.c. }\right\}
$$

while $j_{\mu}^{(-)}$takes a form from which it is manifest that the current of charge does not exist in the case of only one hermitian field;

$$
j_{\mu}^{(-)}(x)=\int_{\Sigma} d \Sigma^{\prime \nu} \frac{1}{2}\left\{W\left(x, x^{\prime}\right) \stackrel{\leftrightarrow}{\partial_{\mu}} \stackrel{\leftrightarrow}{\partial_{\nu}^{\prime}} i\left[\phi_{1}(x) \phi_{2}\left(x^{\prime}\right)-\phi_{2}(x) \phi_{1}\left(x^{\prime}\right)\right]+\text { h.c. }\right\} .
$$

There is one important difference between the currents $j_{\mu}^{(+)}$and $j_{\mu}^{(-)}$. Similarly to the particle current (37), the particle current $j_{\mu}^{(+)}(x)$ possesses the non-local features related to the integration over $\Sigma$ on which $x$ lies. On the other hand, the apparent non-local features of $j_{\mu}^{(-)}(x)$ really do not exist, because, by using the canonical commutation relations (108)-(109) and Eqs. (31)-(33), the integration over $d \Sigma^{\prime \nu}$ can be performed. This cannot be done for $j_{\mu}^{(+)}$ because, owing to the different sign, certain terms that cancel in $j_{\mu}^{(-)}$do not cancel in $j_{\mu}^{(+)}$. Applying the commutation relations (108)-(109) to $j_{\mu}^{(-)}$given by (114) such that $\phi^{\dagger}$ always 
comes to the left and $\phi$ to the right, a straightforward calculation that exploits Eqs. (31)-(33) yields

$$
j_{\mu}^{(-)}(x)=i \phi^{\dagger}(x) \stackrel{\leftrightarrow}{\partial_{\mu}} \phi(x)+\int_{\Sigma} d \Sigma^{\prime \nu} W\left(x, x^{\prime}\right) \stackrel{\leftrightarrow}{\partial_{\mu}} \stackrel{\leftrightarrow}{\partial_{\nu}^{\prime}} W\left(x^{\prime}, x\right)
$$

so all non-local features are contained in the second term that does not depend on $\phi$. Similarly, applying the commutation relations such that $\phi^{\dagger}$ always comes to the right and $\phi$ to the left, $j_{\mu}^{(-)}$can be written as

$$
j_{\mu}^{(-)}(x)=-i \phi(x) \overleftrightarrow{\partial_{\mu}} \phi^{\dagger}(x)-\int_{\Sigma} d \Sigma^{\prime \nu} W\left(x^{\prime}, x\right) \stackrel{\leftrightarrow}{\partial_{\mu}} \stackrel{\leftrightarrow}{\partial_{\nu}^{\prime}} W\left(x, x^{\prime}\right)
$$

From the antisymmetry of the operators $\overleftrightarrow{\partial}_{\mu}$ and $\stackrel{\leftrightarrow}{\partial_{\nu}^{\prime}}$, it follows that the integrals appearing in (120) and (121) are equal. Therefore, by summing (120) and (121) and dividing the sum by 2 , we find a purely local expression

$$
j_{\mu}^{(-)}(x)=\frac{i}{2}\left[\phi^{\dagger}(x) \stackrel{\leftrightarrow}{\partial_{\mu}} \phi(x)-\phi(x) \overleftrightarrow{\partial_{\mu}} \phi^{\dagger}(x)\right]
$$

This is the usual form of the charge current, often derived as a Noether current resulting from the global U(1) invariance of the Lagrangian.

Using (110), one can show that (122) is normally ordered, i.e., that

$$
\left\langle 0\left|j_{\mu}^{(-)}\right| 0\right\rangle=0 .
$$

Alternatively, one can prove (123) by using (110), (26), and (20) to show that

$$
\left\langle 0\left|i \phi^{\dagger}(x) \overleftrightarrow{\partial_{\mu}} \phi(x)\right| 0\right\rangle=-\int_{\Sigma} d \Sigma^{\prime \nu} W\left(x, x^{\prime}\right) \overleftrightarrow{\partial_{\mu}} \overleftrightarrow{\partial_{\nu}^{\prime}} W\left(x^{\prime}, x\right)
$$

which implies that $(120)$ can be written as

$$
j_{\mu}^{(-)}=i \phi^{\dagger} \stackrel{\leftrightarrow}{\partial_{\mu}} \phi-\left\langle 0\left|i \phi^{\dagger} \stackrel{\leftrightarrow}{\partial_{\mu}} \phi\right| 0\right\rangle
$$

Using (26), (110), (20), and $\left[a_{k}, b_{k^{\prime}}\right]=0$, the currents (114), (116), and (117) can be written in a form similar to (38). We find

$$
\begin{gathered}
j_{\mu}^{(P)}=i \sum_{k, k^{\prime}} f_{k}^{*} \stackrel{\leftrightarrow}{\partial_{\mu}} f_{k^{\prime}} a_{k}^{\dagger} a_{k^{\prime}}+j_{\mu}^{\text {mix }}, \\
j_{\mu}^{(A)}=i \sum_{k, k^{\prime}} f_{k}^{*} \stackrel{\leftrightarrow}{\partial_{\mu}} f_{k^{\prime}} b_{k}^{\dagger} b_{k^{\prime}}-j_{\mu}^{\text {mix }}, \\
j_{\mu}^{(+)}=i \sum_{k, k^{\prime}} f_{k}^{*} \stackrel{\leftrightarrow}{\partial_{\mu}} f_{k^{\prime}}\left(a_{k}^{\dagger} a_{k^{\prime}}+b_{k}^{\dagger} b_{k^{\prime}}\right), \\
j_{\mu}^{(-)}=i \sum_{k, k^{\prime}} f_{k}^{*} \stackrel{\leftrightarrow}{\partial_{\mu}} f_{k^{\prime}}\left(a_{k}^{\dagger} a_{k^{\prime}}-b_{k}^{\dagger} b_{k^{\prime}}\right)+2 j_{\mu}^{\text {mix }},
\end{gathered}
$$

where the mixed hermitian current $j_{\mu}^{\text {mix }}$ mixes (in products) the particle operators $a_{k}, a_{k}^{\dagger}$ with the antiparticle operators $b_{k}, b_{k}^{\dagger}$ :

$$
j_{\mu}^{\mathrm{mix}}=\frac{i}{2} \sum_{k, k^{\prime}}\left(f_{k} \stackrel{\leftrightarrow}{\partial_{\mu}} f_{k^{\prime}} a_{k^{\prime}} b_{k}+f_{k}^{*} \stackrel{\leftrightarrow}{\partial_{\mu}} f_{k^{\prime}}^{*} a_{k}^{\dagger} b_{k^{\prime}}^{\dagger}\right)
$$


Now we see that

$$
j_{\mu}^{(+)}|0\rangle=0,
$$

while the currents (126), (127), and (129) have not this property. In the case of Minkowski spacetime, Eq. (43) generalizes to

$$
\tilde{J}_{\mu}^{( \pm)} \equiv \int d^{3} x j_{\mu}^{( \pm)}(x)=\sum_{\mathbf{k}} \frac{k_{\mu}}{\omega_{\mathbf{k}}}\left(a_{\mathbf{k}}^{\dagger} a_{\mathbf{k}} \pm b_{\mathbf{k}}^{\dagger} b_{\mathbf{k}}\right)
$$

From $(\sqrt[6]{6})$ and $(\sqrt{29})$ it follows that the currents (114) are conserved:

$$
\nabla^{\mu} j_{\mu}^{( \pm)}=0
$$

When a non-gravitational interaction is described by (64), then $j_{\mu}^{(+)}$satisfies an equation similar to $(69)$, i.e.

$$
\nabla^{\mu} j_{\mu}^{(+)}(x)=\int_{\Sigma} d \Sigma^{\prime \nu} \frac{1}{2}\left\{W\left(x, x^{\prime}\right) \stackrel{\leftrightarrow}{\partial_{\nu}^{\prime}}\left[J^{\dagger}(x) \phi\left(x^{\prime}\right)+J(x) \phi^{\dagger}\left(x^{\prime}\right)\right]+\text { h.c. }\right\}
$$

On the other hand, $j_{\mu}^{(-)}$is conserved again, provided that the Lagrangian possesses a global $\mathrm{U}(1)$ symmetry. The conservation of $j_{\mu}^{(-)}$is related to the fact that the second term in (120) does not depend on the interaction described by $J$.

When a natural choice of the modes $f_{k}$ does not exist, then one can redefine the particle current $j_{\mu}^{(+)}(\sqrt{114})$ by replacing (26) with $(77)$ or $\bar{G}^{+}\left(x, x^{\prime}\right)$ from Sec. 3.8.

\subsection{The generalization to the electromagnetic background}

Let us generalize the definition of currents $j_{\mu}^{( \pm)}$to the case in which a classical electromagnetic background $\bar{A}_{\mu}(x)$ is also present. Under a gauge transformation

$$
\bar{A}_{\mu}^{\prime}(x)=\bar{A}_{\mu}(x)+e^{-1} \partial_{\mu} \lambda(x),
$$

the scalar field transforms as

$$
\phi^{\prime}(x)=\phi(x) e^{-i \lambda(x)}, \quad \phi^{\prime \dagger}(x)=\phi^{\dagger}(x) e^{i \lambda(x)} .
$$

The covariant derivatives

$$
D_{\mu}=\nabla_{\mu}+i e \bar{A}_{\mu}, \quad D_{\mu}^{*}=\nabla_{\mu}-i e \bar{A}_{\mu}
$$

are covariant with respect to both the gauge transformations and the general-coordinate transformations. The quantities $D_{\mu} \phi$ and $D_{\mu}^{*} \phi^{*}$ transform as $\phi$ and $\phi^{*}$ in (136), respectively. The fields $\phi$ and $\phi^{*}$ satisfy the equations of motion

$$
\begin{gathered}
\left(D^{\mu} D_{\mu}+m^{2}+\xi R\right) \phi=0 \\
\left(D^{* \mu} D_{\mu}^{*}+m^{2}+\xi R\right) \phi^{\dagger}=0 .
\end{gathered}
$$

The solution can be expanded as

$$
\begin{aligned}
\phi(x) & =\sum_{k} a_{k} f_{k}(x)+b_{k}^{\dagger} g_{k}^{*}(x), \\
\phi^{\dagger}(x) & =\sum_{k} a_{k}^{\dagger} f_{k}^{*}(x)+b_{k} g_{k}(x) .
\end{aligned}
$$


When $\bar{A}_{\mu}=0$, then $f_{k}=g_{k}$. In general, $f_{k}$ and $g_{k}^{*}$ transform as $\phi$ under a gauge transformation, while $f_{k}^{*}$ and $g_{k}$ transform as $\phi^{\dagger}$. Introducing the "antisymmetric" covariant derivative $\stackrel{\leftrightarrow}{D_{\mu}}$ defined by

$$
a \stackrel{\leftrightarrow}{D}_{\mu} b=a D_{\mu} b-\left(D_{\mu}^{*} a\right) b
$$

we define two different scalar products:

$$
\begin{aligned}
& (a, b)=i \int_{\Sigma} d \Sigma^{\mu} a^{*} \overleftrightarrow{D}_{\mu} b \\
& (a, b)_{*}=i \int_{\Sigma} d \Sigma^{\mu} a^{*} \stackrel{\leftrightarrow}{D_{\mu}^{*}} b
\end{aligned}
$$

These scalar products are gauge invariant, provided that $a$ and $b$ transform in the same way under the gauge transformation, i.e., either as $\phi$ or as $\phi^{\dagger}$. When $a$ commutes with $b$, then

$$
a \stackrel{\leftrightarrow}{D}_{\mu}^{*} b=-b \stackrel{\leftrightarrow}{D}_{\mu} a
$$

Consequently, in the commuting case, the two scalar products are related as

$$
(a, b)_{*}=-\left(b^{*}, a^{*}\right)
$$

The modes $f_{k}$ and $g_{k}$ satisfy

$$
\begin{gathered}
\left(f_{k}, f_{k^{\prime}}\right)=-\left(f_{k}^{*}, f_{k^{\prime}}^{*}\right)_{*}=\delta_{k k^{\prime}}, \\
\left(g_{k}, g_{k^{\prime}}\right)_{*}=-\left(g_{k}^{*}, g_{k^{\prime}}^{*}\right)=\delta_{k k^{\prime}}, \\
\left(f_{k}, g_{k^{\prime}}^{*}\right)=\left(g_{k}, f_{k^{\prime}}^{*}\right)_{*}=0 .
\end{gathered}
$$

Therefore, the operators $a_{k}$ and $b_{k}$ satisfy the usual algebra of lowering and raising operators and can be written as

$$
\begin{gathered}
a_{k}=\left(f_{k}, \phi\right), \quad b_{k}^{\dagger}=-\left(g_{k}^{*}, \phi\right), \\
a_{k}^{\dagger}=-\left(f_{k}^{*}, \phi^{\dagger}\right)_{*}, \quad b_{k}=\left(g_{k}, \phi^{\dagger}\right)_{*} .
\end{gathered}
$$

The vacuum $|0\rangle$ is defined by

$$
a_{k}|0\rangle=b_{k}|0\rangle=0
$$

We introduce two 2-point functions:

$$
\begin{aligned}
& W^{(P)}\left(x, x^{\prime}\right)=\sum_{k} f_{k}(x) f_{k}^{*}\left(x^{\prime}\right), \\
& W^{(A)}\left(x, x^{\prime}\right)=\sum_{k} g_{k}(x) g_{k}^{*}\left(x^{\prime}\right) .
\end{aligned}
$$

From (139) and the fact that the operators $a_{k}$ and $a_{k}^{\dagger}$ are related to particles while $b_{k}$ and $b_{k}^{\dagger}$ are related to antiparticles, we see that $W^{(P)}$ is related to the propagator of particles, while $W^{(A)}$ is related to the propagator of antiparticles. In general, $W^{(P)} \neq W^{(A)}$, which corresponds to the fact that particles and antiparticles propagate in a different way, provided that a background electromagnetic field exists. When $\bar{A}_{\mu}=0$, then $W^{(P)}=W^{(A)}=W$, where $W$ is the 2-point function used in Sec. 4.1. This means that particles and antiparticles propagate in the same way when a background electromagnetic field does not exist. When $\bar{F}_{\mu \nu} \equiv \partial_{\mu} \bar{A}_{\nu}-\partial_{\nu} \bar{A}_{\mu}=0$ but $\bar{A}_{\mu}=e^{-1} \partial_{\mu} \lambda \neq 0$, then

$$
\begin{gathered}
W^{(P)}\left(x, x^{\prime}\right)=e^{-i\left[\lambda(x)-\lambda\left(x^{\prime}\right)\right]} W\left(x, x^{\prime}\right), \\
W^{(A)}\left(x, x^{\prime}\right)=e^{i\left[\lambda(x)-\lambda\left(x^{\prime}\right)\right]} W\left(x, x^{\prime}\right),
\end{gathered}
$$


so the two 2-point functions differ only by a phase, which should not have any physical effects. The functions $W^{(P)}$ and $W^{(A)}$ satisfy

$$
\begin{gathered}
\left(D^{\mu} D_{\mu}+m^{2}+\xi R(x)\right) W^{(P)}\left(x, x^{\prime}\right)=0, \\
\left(D^{\prime * \mu} D_{\mu}^{\prime *}+m^{2}+\xi R\left(x^{\prime}\right)\right) W^{(P)}\left(x, x^{\prime}\right)=0 \\
\left(D^{* \mu} D_{\mu}^{*}+m^{2}+\xi R(x)\right) W^{(A)}\left(x, x^{\prime}\right)=0 \\
\left(D^{\prime \mu} D_{\mu}^{\prime}+m^{2}+\xi R\left(x^{\prime}\right)\right) W^{(A)}\left(x, x^{\prime}\right)=0 .
\end{gathered}
$$

Therefore, $W^{(P)}$ and $W^{(A)}$ transform to each other when $\bar{A}_{\mu}$ transforms to $-\bar{A}_{\mu}$, which corresponds to the transformation $\bar{F}_{\mu \nu} \rightarrow-\bar{F}_{\mu \nu}$. From (139) it follows that $W^{(P)}$ and $W^{(A)}$ are equal to the Wightman functions

$$
\begin{aligned}
& W^{(P)}\left(x, x^{\prime}\right)=\left\langle 0\left|\phi(x) \phi^{\dagger}\left(x^{\prime}\right)\right| 0\right\rangle, \\
& W^{(A)}\left(x, x^{\prime}\right)=\left\langle 0\left|\phi^{\dagger}(x) \phi\left(x^{\prime}\right)\right| 0\right\rangle,
\end{aligned}
$$

while from (147) we find

$$
W^{(P) *}\left(x, x^{\prime}\right)=W^{(P)}\left(x^{\prime}, x\right), \quad W^{(A) *}\left(x, x^{\prime}\right)=W^{(A)}\left(x^{\prime}, x\right),
$$

Under gauge transformations, $W^{(P)}\left(x, x^{\prime}\right)$ transforms as $\phi(x) \phi^{\dagger}\left(x^{\prime}\right)$, while $W^{(A)}\left(x, x^{\prime}\right)$ transforms as $\phi^{\dagger}(x) \phi\left(x^{\prime}\right)$. From the canonical commutation relations

$$
\begin{gathered}
{\left[\phi^{\dagger}(x), \phi\left(x^{\prime}\right)\right]_{\Sigma}=\left[D_{0}^{*} \phi^{\dagger}(x), D_{0}^{\prime} \phi\left(x^{\prime}\right)\right]_{\Sigma}=0,} \\
\tilde{n}^{0}\left(x^{\prime}\right)\left[\phi^{\dagger}(x), D_{0}^{\prime} \phi\left(x^{\prime}\right)\right]_{\Sigma}=i \delta^{3}\left(\mathbf{x}-\mathbf{x}^{\prime}\right),
\end{gathered}
$$

we find that $f_{k}$ and $g_{k}$ are functions such that

$$
\begin{gathered}
{\left[W^{(P)}\left(x, x^{\prime}\right)-W^{(A)}\left(x^{\prime}, x\right)\right]_{\Sigma}=0,} \\
D_{0} D_{0}^{\prime *}\left[W^{(P)}\left(x, x^{\prime}\right)-W^{(A)}\left(x^{\prime}, x\right)\right]_{\Sigma}=0, \\
\tilde{n}^{0}\left(x^{\prime}\right) D_{0}^{\prime *}\left[W^{(P)}\left(x, x^{\prime}\right)-W^{(A)}\left(x^{\prime}, x\right)\right]_{\Sigma}=i \delta^{3}\left(\mathbf{x}-\mathbf{x}^{\prime}\right) .
\end{gathered}
$$

Now we introduce the global quantities:

$$
N^{( \pm)}=\sum_{k} a_{k}^{\dagger} a_{k} \pm b_{k}^{\dagger} b_{k}
$$

Writing this in the covariant form (113), we find

$$
\begin{aligned}
j_{\mu}^{( \pm)}(x)= & \int_{\Sigma} d \Sigma^{\prime \nu} \frac{1}{2}\left\{\left[W^{(P)}\left(x, x^{\prime}\right) \stackrel{\leftrightarrow}{D_{\mu}^{*}} \stackrel{\leftrightarrow}{D_{\nu}^{\prime}} \phi^{\dagger}(x) \phi\left(x^{\prime}\right)\right.\right. \\
& \left.\left. \pm W^{(A)}\left(x, x^{\prime}\right) \stackrel{\leftrightarrow}{D_{\mu}} \stackrel{\leftrightarrow}{D_{\nu}^{\prime *}} \phi(x) \phi^{\dagger}\left(x^{\prime}\right)\right]+ \text { h.c. }\right\}
\end{aligned}
$$

Although $W^{(A)}, W^{(P)}, \phi$, and $\phi^{\dagger}$ depend on gauge, the currents (156) do not depend on gauge. Therefore, our definition of particles and that of charge are gauge invariant. Eq. (156) is the gauge-invariant generalization of (114).

In a way similar to the case $\bar{A}_{\mu}=0$, it can be shown that $j_{\mu}^{(-)}$can be written in a purely local form

$$
\begin{aligned}
j_{\mu}^{(-)} & =i \phi^{\dagger} \stackrel{\leftrightarrow}{D_{\mu}} \phi-\left\langle 0\left|i \phi^{\dagger} \stackrel{\leftrightarrow}{D_{\mu}} \phi\right| 0\right\rangle \\
& =-i \phi \stackrel{\leftrightarrow}{D_{\mu}^{*}} \phi^{\dagger}+\left\langle 0\left|i \phi \overleftrightarrow{D_{\mu}^{*}} \phi^{\dagger}\right| 0\right\rangle
\end{aligned}
$$


This is the usual form of the charge current, often derived from the gauge invariance of the Lagrangian.

From (149) and (138) it follows that both currents are conserved:

$$
\nabla^{\mu} j_{\mu}^{( \pm)}=0
$$

Of course, the conservation of the charge current $j_{\mu}^{(-)}$was expected because it is well known that (157) is conserved. However, the conservation of $j_{\mu}^{(+)}$is less trivial because it implies that the background electromagnetic field described by $\bar{A}_{\mu}$ does not produce particles (particleantiparticle pairs), provided that a unique (or a preferred) vacuum defined by (146) exists.

In general, a natural definition of the vacuum (146) does not exist because the natural choice of the modes $f_{k}$ and $g_{k}$ does not exist. In this case, one can introduce different modes that are natural at different times, similarly as in Sec. 3.7. In a time-dependent background $\bar{A}_{\mu}$, the natural modes at a given time can be chosen such that they are positive-frequency modes at that time 13]. Alternatively, in a time-independent but space-dependent background $\bar{A}_{\mu}$, one can introduce "in" and "out" modes, the naturalness of which is related to the sign of the wave vector $\mathbf{k}$ in asymptotic regions [35]. In this way, one can describe the pair creation by the electromagnetic background in a local way, similarly as in Sec. 3.7. As in the case of particle creation by the gravitational background studied in Sec. 3.7, it is easy to see that this local description of pair creation is equivalent to the usual global description based on the Bogoliubov transformation.

However, the sign of the frequency and that of the wave vector are not gauge-invariant concepts. In some cases, such as $\bar{F}_{\mu \nu}=0$ or a time-independent background electric field, some gauges may seem "natural", but a general recipe for choosing the gauge does not exist. Therefore, by using the Bogoliubov-transformation method, it is not possible, even in principle, to calculate the distribution of produced pairs in an arbitrary electromagnetic background.

The following remark also illustrates why the Bogoliubov-transformation method is problematic. For $\bar{F}_{\mu \nu}=0$, the "natural" gauge is $\bar{A}_{\mu}=0$, which defines the "natural" vacuum. However, by using a different gauge, one can obtain that this vacuum is a many-particle state. In particular, an appropriate gauge leads to a thermal distribution of particles in the "natural" vacuum, leading to an electromagnetic analog of the Rindler quantization and the Unruh effect. On the other hand, physical quantities should not depend on gauge, which again raises doubts about the appropriateness of the Bogoliubov-transformation method.

Now we see that just as the particle current based on $(77)$ is not really general-covariant, a similar modification of (156) is not really gauge-invariant. To define particles in a unique way, one needs a universal natural choice of the functions $W^{(P)}$ and $W^{(A)}$ using a method that does not require a particular choice of the modes $f_{k}$ and $g_{k}$. Again, such a choice exists and is a generalization of the choice in Sec. 3.8. By introducing the Feynman Green functions $G_{F}^{(P)}$ and $G_{F}^{(A)}$ calculated by using Schwinger's method [15, 10, 11, 36], we propose

$$
W^{(P)}\left(x, x^{\prime}\right)=G^{(P)+}\left(x, x^{\prime}\right), \quad W^{(A)}\left(x, x^{\prime}\right)=G^{(A)+}\left(x, x^{\prime}\right) .
$$

(Note that the Green functions are calculated for Minkowski spacetime in the references cited above, but the formalism can be generalized to curved spacetime as well.) Here $G^{(P)+}$ and $G^{(A)+}$ satisfy (149), i.e.

$$
\begin{gathered}
\left(D^{\mu} D_{\mu}+m^{2}+\xi R(x)\right) G^{(P)+}\left(x, x^{\prime}\right)=0, \\
\left(D^{\prime * \mu} D_{\mu}^{\prime *}+m^{2}+\xi R\left(x^{\prime}\right)\right) G^{(P)+}\left(x, x^{\prime}\right)=0, \\
\left(D^{* \mu} D_{\mu}^{*}+m^{2}+\xi R(x)\right) G^{(A)+}\left(x, x^{\prime}\right)=0, \\
\left(D^{\prime \mu} D_{\mu}^{\prime}+m^{2}+\xi R\left(x^{\prime}\right)\right) G^{(A)+}\left(x, x^{\prime}\right)=0,
\end{gathered}
$$


and are calculated from the corresponding Feynman Green functions that satisfy similar equations, but with the right-hand sides proportional to $\delta^{4}\left(x-x^{\prime}\right)$. Note that in the literature 15, 10, 11, 36], usually only one Feynman Green function corresponding to $G_{F}^{(P)}$ is considered and is called simply $G_{F}$. However, there are actually two Feynman Green functions $G_{F}^{(P)}$ and $G_{F}^{(A)}$ that satisfy similar equations, but with $\bar{A}_{\mu}$ replaced with $-\bar{A}_{\mu}$.

From (159), (160), and (138) it follows that the currents (156) are conserved. In particular, this implies that the most natural definition of the particle current in an electromagnetic background leads to the result that classical electromagnetic backgrounds do not produce particles. This is in agreement with some other results [26, 27, 28]. It is interesting to note that a different gauge-invariant formalism based on the same Green function $G_{F}$ as above leads to a result that a classical background static electric field produces particle-antiparticle pairs [15]. However, when the formalism of [15] is naturally modified so that the integration contour over a scalar variable remains on the real axis (see (86)), then this formalism does not lead to pair production. As argued in 28], such a modified formalism might be the correct one.

\subsection{The generalization to the quantum electromagnetic interaction}

As we have seen, the result that the interaction with a classical electromagnetic background does not lead to a change of the number of particles is closely related to gauge invariance. However, it is known from many experiments that the electromagnetic interaction may cause a change of the number of charged particles. The best known example is the annihilation of a particleantiparticle pair to a pair of photons. Such processes are successfully described by Feynman diagrams that result from a theory in which the electromagnetic field is also quantized. Physical arguments explaining why only a quantum electromagnetic interaction can consistently describe a change of the number of particles are given in [28]. Here we give a formal explanation of this.

Let $\hat{A}_{\mu}$ be the total operator of the electromagnetic field. It can be written as

$$
\hat{A}_{\mu}=A_{\mu}+\bar{A}_{\mu}
$$

where $A_{\mu}$ is the operator such that

$$
\left\langle 0\left|A_{\mu}\right| 0\right\rangle=0
$$

from which it follows that

$$
\left\langle 0\left|\hat{A}_{\mu}\right| 0\right\rangle=\bar{A}_{\mu} .
$$

When $A_{\mu}$ does not interact with other fields, then it can be expanded in the usual way in terms of creation and annihilation operators. A gauge transformation

$$
\hat{A}_{\mu}^{\prime}=\hat{A}_{\mu}+e^{-1} \partial_{\mu} \lambda
$$

where $\lambda(x)$ is a c-number function, can be written as

$$
\begin{gathered}
A_{\mu}^{\prime}=A_{\mu}, \\
\bar{A}_{\mu}^{\prime}=\bar{A}_{\mu}+e^{-1} \partial_{\mu} \lambda .
\end{gathered}
$$

Therefore, the operator $A_{\mu}$ is invariant with respect to the classical gauge transformations (165). The generalization of (137) is

$$
\hat{D}_{\mu}=\nabla_{\mu}+i e \hat{A}_{\mu}=D_{\mu}+i e A_{\mu} .
$$


The currents $j_{\mu}^{( \pm)}$take the same form (156) with the same covariant derivative $\stackrel{\leftrightarrow}{D}_{\mu}$ and with the same functions $W^{(P)}$ and $W^{(A)}$ satisfying Eqs. (149). However, in these currents, the field $\phi$ satisfies

$$
\left(\hat{D}^{\mu} \hat{D}_{\mu}+m^{2}+\xi R\right) \phi=0
$$

and similarly for $\phi^{\dagger}$. Eq. (167) can also be written in a form similar to (64):

$$
\left(D^{\mu} D_{\mu}+m^{2}+\xi R\right) \phi=J
$$

where

$$
J=\left[e^{2} A^{\mu} A_{\mu}-i e\left(\nabla_{\mu} A^{\mu}\right)\right] \phi-2 i e A^{\mu} D_{\mu} \phi .
$$

We see that $J$ transforms under the gauge transformations (165) in the same way as $\phi$, so (168) is covariant. Similarly, the currents $j_{\mu}^{( \pm)}$given by $(156)$ are invariant with respect to gauge transformations (165). The local particle production is described by the gauge-invariant generalization of (134). In a similar way as before, it can be shown that this local particle production is consistent with the particle production described in the usual way using the interaction picture, in which the interaction Hamiltonian is the part of the Hamiltonian that generates the right-hand side of (168).

The conservation of charge is a little bit tricky. One can introduce two charge currents:

$$
q_{\mu}=i \phi^{\dagger} \stackrel{\leftrightarrow}{D}_{\mu} \phi, \quad \hat{q}_{\mu}=i \phi^{\dagger} \stackrel{\leftrightarrow}{D}_{\mu} \phi
$$

Again, it can be shown that

$$
j_{\mu}^{(-)}=q_{\mu}-\left\langle 0\left|q_{\mu}\right| 0\right\rangle=: q_{\mu}:
$$

However, this current is not conserved. From the equation of motion (167) it follows that the current $\hat{q}_{\mu}$ is conserved, while $q_{\mu}$ is not:

$$
\begin{gathered}
\nabla^{\mu} \hat{q}_{\mu}=0, \\
\nabla^{\mu} q_{\mu}=2 e\left[\left(\nabla_{\mu} A^{\mu}\right) \phi^{\dagger} \phi+A^{\mu} \nabla_{\mu}\left(\phi^{\dagger} \phi\right)\right] .
\end{gathered}
$$

Note that the right-hand sides in (172) do not depend on the background $\bar{A}_{\mu}$, so the following peculiarities appear even when $\bar{A}_{\mu}=0$. The two currents are related as

$$
\hat{q}_{\mu}=q_{\mu}-2 e A_{\mu} \phi^{\dagger} \phi .
$$

Which charge current is more physical, $\hat{q}_{\mu}$ or $q_{\mu}$ ? It depends on how the charge is measured. If the charge is measured so that the corresponding electromagnetic effects determined by the total electromagnetic field $\hat{F}_{\mu \nu}=\partial_{\mu} \hat{A}_{\nu}-\partial_{\nu} \hat{A}_{\mu}$ are measured, then the current $\hat{q}_{\mu}$ is the physical one, because the Maxwell equations read

$$
\nabla^{\nu} \hat{F}_{\mu \nu}=-e: \hat{q}_{\mu}:
$$

However, this does not mean that the current $q_{\mu}$ is unphysical. Each hermitian operator, conserved or not, is an observable. In order to understand the physical meaning of $q_{\mu}$, in the rest of this subsection we work in the interaction picture, in which the field $\phi$ has the expansion (139) and $A_{\mu}$ has a similar expansion in terms of creation and annihilation operators. If one simply counts the number of particles and the number of antiparticles, then the current $q_{\mu}$ is the physical one because the corresponding charge

$$
Q=\int_{\Sigma} d \Sigma^{\mu}: q_{\mu}:=N^{(-)} \equiv \sum_{k} a_{k}^{\dagger} a_{k}-b_{k}^{\dagger} b_{k}
$$


is equal to the difference of these two numbers. On the other hand, the conserved charge

$$
\hat{Q}=\int_{\Sigma} d \Sigma^{\mu}: \hat{q}_{\mu}:=N^{(-)}-2 e \int_{\Sigma} d \Sigma^{\mu} A_{\mu}: \phi^{\dagger} \phi:
$$

depends not only on the number of charged particles, but also on the number of photons.

An interesting question is the following. Can one observe, by counting the number of scalar particles and antiparticles, that the charge $Q$ is not conserved? Since the extra term in (173) is linear in $A_{\mu}$, the expected value of this operator vanishes for any state in which the number of photons is definite (i.e., the state is not a superposition of states with different numbers of photons). Therefore, the expected values of the charges $Q$ and $\hat{Q}$ are equal for such states, so the charge $Q$ is conserved in this case. Note also that the number of any kind of particles is definite in any particular Feynman diagram, so the charge $Q$ is also conserved on the level of Feynman diagrams. The conservation of $Q$ on the level of Feynman diagrams can also be seen directly from the fact that, in the interaction Hamiltonian, the fields $\phi$ and $\phi^{\dagger}$ always appear in

products of the form $\phi^{\dagger} \phi$ and $\phi^{\dagger} \overleftrightarrow{D}_{\mu} \phi$. However, if the initial state $|i\rangle$ is a state with a definite number of all kinds of particles, then the final state determined by the $S$-matrix operator takes the form

$$
|f\rangle=S|i\rangle=\sum_{n} C_{i f_{n}}\left|f_{n}\right\rangle .
$$

Here the states $\left|f_{n}\right\rangle$ have a definite number of all kinds of particles, but the total state $|f\rangle$ has not. (The coefficients $C_{i f_{n}}=\left\langle f_{n} \mid f\right\rangle=\left\langle f_{n}|S| i\right\rangle$ are the amplitudes determined by the corresponding Feynman diagrams.) Therefore, it is possible, in principle, to find the system in a state in which the final charge $Q$ is not equal to the initial charge $Q$. However, it seems very unlikely that this will happen in practice because $Q$ is conserved in any experiment in which the state $|f\rangle$ collapses to a state with a definite number of particles, or the decoherence destroys all mixing terms so that the observable degrees of freedom can be described by a density matrix that is not a pure state, but a classical mixture of the states with different numbers of particles.

Note, finally, that analogous problems with the conservation of charge do not exist for spin$1 / 2$ fields. In the Standard Model, fundamental charged scalar fields do not exist. There is the complex Higgs field, but, after the spontaneous symmetry breaking, it reduces to an uncharged hermitian Higgs field. Therefore, the problems with the conservation of charge for complex scalar fields may be phenomenologically irrelevant.

\section{Spinor field}

For simplicity, we study the spinor fields in Minkowski spacetime. However, all equations can be generalized to an arbitrary spacetime by replacing the derivatives $\partial_{\mu}$ with the general-covariant derivatives and by replacing certain constant Dirac matrices with the $x$-dependent Dirac matrices $\gamma_{\mu}(x)=e_{\mu}^{\alpha}(x) \gamma_{\alpha}$, where $e_{\mu}^{\alpha}(x)$ is the vierbein [2]. When appropriate, we shortly discuss the effects of this generalization.

\subsection{Particle and charge currents}

The Dirac matrices satisfy the anticommutation relations

$$
\left\{\gamma_{\mu}, \gamma_{\nu}\right\}=2 \eta_{\mu \nu}
$$

We choose their representation such that 19

$$
\gamma_{\mu}^{\dagger}=\gamma_{0} \gamma_{\mu} \gamma_{0} .
$$


The massive spin- $1 / 2$ field $\psi(x)$ satisfies the Dirac equation

$$
(i \not \partial-m) \psi=0, \quad \bar{\psi}(i \overleftarrow{\not \supset}+m)=0
$$

where $\not \partial=\gamma_{\mu} \partial^{\mu}$ and $\bar{\psi}=\psi^{\dagger} \gamma_{0}$. The field can be expanded as

$$
\begin{aligned}
& \psi(x)=\sum_{k} b_{k} u_{k}(x)+d_{k}^{\dagger} v_{k}(x), \\
& \bar{\psi}(x)=\sum_{k} b_{k}^{\dagger} \bar{u}_{k}(x)+d_{k} \bar{v}_{k}(x),
\end{aligned}
$$

where the summation over $k$ includes the summation over spin indices as well. The scalar product is

$$
(\psi, \chi)=\int_{\Sigma} d \Sigma^{\mu} \bar{\psi} \gamma_{\mu} \chi
$$

The spinors $u_{k}$ and $v_{k}$ satisfy

$$
\begin{gathered}
\left(u_{k}, u_{k^{\prime}}\right)=\left(v_{k}, v_{k^{\prime}}\right)=\delta_{k k^{\prime}} \\
\left(u_{k}, v_{k^{\prime}}\right)=\left(v_{k}, u_{k^{\prime}}\right)=0
\end{gathered}
$$

Therefore,

$$
\begin{aligned}
& b_{k}=\left(u_{k}, \psi\right), \quad d_{k}^{\dagger}=\left(v_{k}, \psi\right), \\
& b_{k}^{\dagger}=\left(\psi, u_{k}\right), \quad d_{k}=\left(\psi, v_{k}\right) .
\end{aligned}
$$

The canonical anticommutation relations can be written as

$$
\begin{gathered}
\left\{\psi_{a}(x), \psi_{b}\left(x^{\prime}\right)\right\}_{\Sigma}=\left\{\left(\bar{\psi}(x) \gamma_{0}\right)_{a},\left(\bar{\psi}\left(x^{\prime}\right) \gamma_{0}\right)_{b}\right\}_{\Sigma}=0 \\
\left.\left\{\psi_{a}(x), \bar{\psi}_{c}\left(x^{\prime}\right)\right\}\left(\gamma_{0}\right)_{c b}\right|_{\Sigma}=\delta_{a b} \delta^{3}\left(\mathbf{x}-\mathbf{x}^{\prime}\right) .
\end{gathered}
$$

From (185) it follows that the operators (184) satisfy the usual algebra of fermion creation and annihilation operators:

$$
\left\{b_{k}, b_{k^{\prime}}^{\dagger}\right\}=\left\{d_{k}, d_{k^{\prime}}^{\dagger}\right\}=\delta_{k k^{\prime}},
$$

while other anticommutators vanish. We introduce the particle and antiparticle 2-point functions

$$
\begin{aligned}
& S^{(P)}\left(x, x^{\prime}\right)=\sum_{k} u_{k}(x) \bar{u}_{k}\left(x^{\prime}\right), \\
& S^{(A)}\left(x, x^{\prime}\right)=\sum_{k} v_{k}\left(x^{\prime}\right) \bar{v}_{k}(x) .
\end{aligned}
$$

They satisfy

$$
\begin{aligned}
& (i \not \partial-m) S^{(P)}\left(x, x^{\prime}\right)=0, \quad S^{(P)}\left(x, x^{\prime}\right)\left(i \overleftarrow{\not \partial^{\prime}}+m\right)=0 \\
& S^{(A)}\left(x, x^{\prime}\right)(i \overleftarrow{\not \supset}+m)=0, \quad\left(i \not \not^{\prime}-m\right) S^{(A)}\left(x, x^{\prime}\right)=0
\end{aligned}
$$

and have the property

$$
\begin{aligned}
\left(S^{(P)}\left(x, x^{\prime}\right)\right)_{a b} & =\left\langle 0\left|\psi_{a}(x) \bar{\psi}_{b}\left(x^{\prime}\right)\right| 0\right\rangle \\
\left(S^{(A)}\left(x, x^{\prime}\right)\right)_{a b} & =\left\langle 0\left|\bar{\psi}_{b}(x) \psi_{a}\left(x^{\prime}\right)\right| 0\right\rangle .
\end{aligned}
$$


We also introduce the 2-point functions $S_{\mu \nu}^{(P)}\left(x, x^{\prime}\right)$ and $S_{\mu \nu}^{(A)}\left(x, x^{\prime}\right)$ defined as

$$
S_{\mu \nu}^{(P, A)}\left(x, x^{\prime}\right)=\gamma_{\mu} S^{(P, A)}\left(x, x^{\prime}\right) \gamma_{\nu}
$$

Using (178), (179) and (187) we find

$$
\gamma_{0} S^{(P, A) \dagger}\left(x, x^{\prime}\right) \gamma_{0}=S^{(P, A)}\left(x^{\prime}, x\right)
$$

while from (178), (179), (185), and (189) it follows that

$$
\begin{gathered}
{\left[S^{(P)}\left(x, x^{\prime}\right)-S^{(A)}\left(x^{\prime}, x\right)\right]_{\Sigma}=\gamma_{0} \delta^{3}\left(\mathbf{x}-\mathbf{x}^{\prime}\right),} \\
{\left[S_{\mu \nu}^{(P)}\left(x, x^{\prime}\right)-S_{\mu \nu}^{(A)}\left(x^{\prime}, x\right)\right]_{\Sigma}=\gamma_{\mu} \gamma_{0} \gamma_{\nu} \delta^{3}\left(\mathbf{x}-\mathbf{x}^{\prime}\right),} \\
{\left[S_{\mu 0}^{(P)}\left(x, x^{\prime}\right)-S_{\mu 0}^{(A)}\left(x^{\prime}, x\right)\right]_{\Sigma}=\gamma_{\mu} \delta^{3}\left(\mathbf{x}-\mathbf{x}^{\prime}\right) .}
\end{gathered}
$$

The global quantities

$$
N^{( \pm)}=\sum_{k} b_{k}^{\dagger} b_{k} \pm d_{k}^{\dagger} d_{k}
$$

can be written in the covariant form as

$$
N^{( \pm)}=\int_{\Sigma} d \Sigma^{\mu} j_{\mu}^{( \pm)}
$$

where

$$
\begin{aligned}
j_{\mu}^{( \pm)}(x)= & \int_{\Sigma} d \Sigma^{\prime \nu} \frac{1}{2}\left\{\left[\bar{\psi}(x) S_{\mu \nu}^{(P)}\left(x, x^{\prime}\right) \psi\left(x^{\prime}\right)\right.\right. \\
& \left.\left. \pm \psi^{T}(x) S_{\nu \mu}^{(A) T}\left(x, x^{\prime}\right) \bar{\psi}^{T}\left(x^{\prime}\right)\right]+ \text { h.c. }\right\}
\end{aligned}
$$

and the superscript $T$ denotes the transpose. From (180) and (188) it follows that both currents are conserved:

$$
\partial^{\mu} j_{\mu}^{( \pm)}=0
$$

By an appropriate generalization to a curved background, it is straightforward to see that the currents $j_{\mu}^{( \pm)}$are conserved again. Also, one can introduce different natural spinors at different times, which redefines the 2-point functions (187) and leads to a local description of particle production by the gravitational background consistent with the Bogoliubov transformation method. The most natural choice of the 2-point functions is

$$
\begin{aligned}
S^{(P)}\left(x, x^{\prime}\right) & =(i \not \nabla+m) \bar{G}^{+}\left(x, x^{\prime}\right), \\
S^{(A)}\left(x, x^{\prime}\right) & =\bar{G}^{+}\left(x, x^{\prime}\right)(i \overleftarrow{\not}-m)
\end{aligned}
$$

where $\bar{G}^{+}\left(x, x^{\prime}\right)$ is the 2-point function for scalar fields with $\xi=1 / 4$ [2] discussed in Sec. 3.8. This natural choice also leads to the conservation of the particle current.

Using (185) and (192) we find the standard form of the charge current

$$
j_{\mu}^{(-)}(x)=\bar{\psi}(x) \gamma_{\mu} \psi(x)-\operatorname{Tr} S^{(A)}(x, x) \gamma_{\mu},
$$

which, after using (181) and (187), can be written as

$$
j_{\mu}^{(-)}(x)=\bar{\psi}(x) \gamma_{\mu} \psi(x)-\left\langle 0\left|\bar{\psi}(x) \gamma_{\mu} \psi(x)\right| 0\right\rangle .
$$

The currents $j_{\mu}^{( \pm)}$can also be written as

$$
j_{\mu}^{( \pm)}=j_{\mu}^{(P)} \pm j_{\mu}^{(A)} .
$$


In terms of the creation and annihilation operators, the currents can be written as

$$
\begin{gathered}
j_{\mu}^{(P)}=\sum_{k, k^{\prime}} \bar{u}_{k^{\prime}} \gamma_{\mu} u_{k} b_{k^{\prime}}^{\dagger} b_{k}+j_{\mu}^{\operatorname{mix}}, \\
j_{\mu}^{(A)}=\sum_{k, k^{\prime}} \bar{v}_{k} \gamma_{\mu} v_{k^{\prime}} d_{k^{\prime}}^{\dagger} d_{k}-j_{\mu}^{\operatorname{mix}}, \\
j_{\mu}^{(+)}=\sum_{k, k^{\prime}} \bar{u}_{k^{\prime}} \gamma_{\mu} u_{k} b_{k^{\prime}}^{\dagger} b_{k}+\bar{v}_{k} \gamma_{\mu} v_{k^{\prime}} d_{k^{\prime}}^{\dagger} d_{k}, \\
j_{\mu}^{(-)}=\sum_{k, k^{\prime}}\left(\bar{u}_{k^{\prime}} \gamma_{\mu} u_{k} b_{k^{\prime}}^{\dagger} b_{k}-\bar{v}_{k} \gamma_{\mu} v_{k^{\prime}} d_{k^{\prime}}^{\dagger} d_{k}\right)+2 j_{\mu}^{\text {mix }},
\end{gathered}
$$

where

$$
j_{\mu}^{\operatorname{mix}}=\frac{1}{2} \sum_{k, k^{\prime}}\left(\bar{v}_{k} \gamma_{\mu} u_{k^{\prime}} d_{k} b_{k^{\prime}}+\bar{u}_{k^{\prime}} \gamma_{\mu} v_{k} b_{k^{\prime}}^{\dagger} d_{k}^{\dagger}\right)
$$

\subsection{The generalization to the electromagnetic interaction}

The expressions for the currents in Sec. 5.1 are already gauge invariant. This makes the generalization to the case of electromagnetic interaction easier than for the scalar fields. This is related to the fact that the Dirac equation is a first-order equation, so the expressions for the currents contain $\gamma_{\mu}$ instead of $\partial_{\mu}$. Consequently, in the currents, one does not need to replace

the derivatives with the gauge-covariant derivatives. Therefore, the currents $j_{\mu}^{( \pm)}$are given by the expression (195). However, the fields satisfy

$$
(i \hat{D}-m) \psi=0, \quad \bar{\psi}\left(i \hat{D}^{*}+m\right)=0
$$

while the 2-point functions satisfy

$$
\begin{aligned}
& (i \not D-m) S^{(P)}\left(x, x^{\prime}\right)=0, \quad S^{(P)}\left(x, x^{\prime}\right)\left(i \overleftarrow{\not D^{\prime *}}+m\right)=0 \\
& S^{(A)}\left(x, x^{\prime}\right)\left(i \overleftarrow{D^{*}}+m\right)=0, \quad\left(i \not D^{\prime}-m\right) S^{(A)}\left(x, x^{\prime}\right)=0
\end{aligned}
$$

The charge current can be written in the form (199) again and is conserved. On the other hand, the particle current is not conserved:

$$
\begin{aligned}
\partial^{\mu} j_{\mu}^{(+)}(x)= & \int_{\Sigma} d \Sigma^{\prime \nu} \frac{1}{2}\left\{i e A _ { \mu } ( x ) \left[\bar{\psi}(x) S_{\mu \nu}^{(P)}\left(x, x^{\prime}\right) \psi\left(x^{\prime}\right)\right.\right. \\
& \left.\left.-\psi^{T}(x) S_{\nu \mu}^{(A) T}\left(x, x^{\prime}\right) \bar{\psi}^{T}\left(x^{\prime}\right)\right]+ \text { h.c. }\right\} .
\end{aligned}
$$

The background $\bar{A}_{\mu}$ does not appear in (208), which means that the background electromagnetic field does not produce particles. Again, it may be interpreted as a consequence of the existence of the preferred spinors $v_{k}$ and $u_{k}$, or as a consequence of the existence of the natural 2-point functions calculated by using Schwinger's method [15, 36]. The particle production by the electromagnetic background can be described by introducing different natural spinors at different times, which redefines the 2-point functions and leads to a local description of particle production consistent with the Bogoliubov transformation method. The particle production described by (208) is equivalent to the usual description based on the interaction picture. 


\section{Conclusion and outlook}

In this paper, the currents of particles and charge for scalar and spinor fields in gravitational and electromagnetic backgrounds have been constructed. The currents are covariant with respect to general coordinate transformations and invariant with respect to gauge transformations. The currents of charge constructed using our method are equal to the usual charge currents. However, previous definitions of particles in quantum field theory were not general-covariant and gaugeinvariant. This is because previous definitions of particles were not local, i.e., the operator of the local particle density was not known, except for the non-relativistic case.

For a given background, the particle current is not unique, but depends on the choice of a 2-point function. Different choices correspond to different definitions of particles. There are 3 types of this choice. The first type is based on the choice of a particular set of complete orthonormal modes that satisfy the field equations of motion. The resulting particle currents give a local description of the particle content in various inequivalent representations of field algebra. The second type is based on choosing different natural modes at different times. This leads to a local description of particle production by classical backgrounds. When the total number of particles on a Cauchy hypersurface is calculated, then the first and second types of choice lead to two types of conventional global concepts of particles. However, the third type, based on the 2-point function calculated using Schwinger's method, is novel even on the global level. This type seems to be the most natural one. It is tempting to interpret these particles as real particles. If this interpretation is correct, then classical backgrounds do not produce real particles. There are also other indications that classical backgrounds might not produce particles [13, 25, 26, 27, 28]. Even if these particles do not correspond to real physical particles in general, it is interesting to ask about the physical meaning of this hermitian observable that corresponds to physical particles at least in Minkowski spacetime and zero electromagnetic background.

As by-products, relevant even without our novel description of particles, the following results have also been obtained. A unique Green function $\bar{G}\left(x, x^{\prime}\right)$ has been constructed for the case in which $x$ and $x^{\prime}$ can be connected by more than one geodesic. It has been found that the requirement of covariance of the time evolution leads to a preferred role of Gaussian coordinates. The peculiarities related to the concept of charge and its conservation in scalar QED have been clarified.

In this paper, the phenomenological applications of the developed formalism have not been studied, but we hope that our results will motivate further investigations. We suggest several possible applications.

First, one can adopt the variant of the formalism according to which background gravitational and electromagnetic fields do produce particles. Contrary to the previous methods, our method enables one to calculate where particles are created. This question is particularly interesting in the case of particle production by black holes. Our formalism is able to test the usual conjecture that particles are created near the horizon.

Second, one can adopt a variant of the formalism according to which background gravitational and electromagnetic fields do not produce particles. It seems very likely that this is the correct interpretation because it results from the definition of particles that seems to be the most natural. In order to calculate particle currents or the total number of particles on a spacelike Cauchy hypersurface, one has to calculate 2-point functions explicitly by using the Schwinger-DeWitt method, which is a non-trivial task in general.

Third, one can study possible physical implications of the fact that the local particle density may be negative. It would be interesting to study more thoroughly how the appearance of negative particle densities depends on the state, entanglement and background interactions, as well as how negative densities disappear by the wave-function collapse or decoherence. For ex- 
ample, the appearance of negative particle densities could be related to the existence of classical "barriers" such as a horizon or an electric potential higher than $2 m$. Eq. (38) suggests that negative particle densities may be related to positive-norm solutions that contain both the positive and the negative frequencies, but this depends on which of the 3 types of 2-point functions is adopted.

In any case, we believe that our results could influence further research that could result in a significant change of the understanding of the concept of particles in quantum field theory. Our local approach changes the notion of a relativistic particle even for a free particle in Minkowski spacetime and zero electromagnetic background.

\section{Acknowledgments}

The author is grateful to S. A. Fulling for a discussion, especially on the possible preferred role of Gaussian coordinates in curved-spacetime quantum field theory. This work was supported by the Ministry of Science and Technology of the Republic of Croatia under Contract No. 0098002.

\section{A Particle density in non-relativistic quantum field theory}

In non-relativistic quantum field theory, the operator of particle density is a well-known quantity [37, 38]. This is a non-negative operator

$$
n(x)=\Psi^{\dagger}(x) \Psi(x)
$$

where $x=(\mathbf{x}, t)$ and $\Psi$ satisfies the Schrödinger equation

$$
-\frac{1}{2 m} \nabla^{2} \Psi+U \Psi=i \frac{\partial \Psi}{\partial t}
$$

with $U(x)$ being a non-relativistic potential. In this appendix, we rederive (209) using the method employed in this paper.

Eq. (210) can be derived from the Lagrangian density

$$
\mathcal{L}=i \Psi^{\dagger} \frac{\partial \Psi}{\partial t}-\frac{1}{2 m}\left(\nabla \Psi^{\dagger}\right)(\nabla \Psi)-U \Psi^{\dagger} \Psi
$$

Therefore, the canonical momentum is $\pi=i \Psi^{\dagger}$ and the canonical equal-time commutation relation between $\Psi$ and $\pi$ can be written as

$$
\left[\Psi(\mathbf{x}, t), \Psi^{\dagger}\left(\mathbf{x}^{\prime}, t\right)\right]=\delta^{3}\left(\mathbf{x}-\mathbf{x}^{\prime}\right) .
$$

The scalar product $\left(\phi_{1}, \phi_{2}\right)=\int d^{3} x \phi_{1}^{*} \phi_{2}$ does not depend on time, provided that $\phi_{1}$ and $\phi_{2}$ satisfy (210). The fields $\Psi$ and $\Psi^{\dagger}$ have the expansion

$$
\Psi(x)=\sum_{k} a_{k} u_{k}(x), \quad \Psi^{\dagger}(x)=\sum_{k} a_{k}^{\dagger} u_{k}^{*}(x)
$$

The modes $u_{k}$ are orthogonal:

$$
\left(u_{k}, u_{k^{\prime}}\right)=\left(u_{k}^{*}, u_{k^{\prime}}^{*}\right)=\delta_{k k^{\prime}}
$$

From (213) and (214) we find

$$
a_{k}=\left(u_{k}, \Psi\right), \quad a_{k}^{\dagger}=\left(u_{k}^{*}, \Psi^{\dagger}\right) .
$$


From the equal-time commutation relations it follows that $a_{k}$ and $a_{k}^{\dagger}$ satisfy the usual algebra of lowering and raising operators [37], so the operator

$$
N=\sum_{k} a_{k}^{\dagger} a_{k}
$$

is the operator of the number of particles. From (213) we see that

$$
\left\langle 0\left|\Psi^{\dagger}(x) \Psi\left(x^{\prime}\right)\right| 0\right\rangle=0, \quad\left\langle 0\left|\Psi(x) \Psi^{\dagger}\left(x^{\prime}\right)\right| 0\right\rangle=W\left(x, x^{\prime}\right),
$$

where

$$
W\left(x, x^{\prime}\right)=\sum_{k} u_{k}(x) u_{k}^{*}\left(x^{\prime}\right) .
$$

From (212), (217), and (218) we see that the modes $u_{k}$ are complete, i.e., that

$$
W\left((\mathbf{x}, t),\left(\mathbf{x}^{\prime}, t\right)\right)=\delta^{3}\left(\mathbf{x}-\mathbf{x}^{\prime}\right) .
$$

Using (215) and (218), we can write (216) as

$$
N=\int d^{3} x n(x)
$$

where

$$
n(x)=\int d^{3} x^{\prime} \frac{1}{2}\left\{W\left(x, x^{\prime}\right) \Psi^{\dagger}(x) \Psi\left(x^{\prime}\right)+\text { h.c. }\right\} .
$$

In (221), $t=t^{\prime}$, so (221) and (219) lead to (209). Therefore, contrary to the relativistic case, in the non-relativistic case the particle density is non-negative and can be written in a purely local form.

For comparison with the relativistic case, let us study the free case $U=0$. The modes $u_{k}$ can be chosen to be the plane-wave modes $u_{\mathbf{k}}$, which, in a finite volume $V$, are

$$
u_{\mathbf{k}}(\mathbf{x}, t)=\frac{e^{-i\left(E_{\mathbf{k}} t-\mathbf{k x}\right)}}{\sqrt{V}}
$$

where $E_{\mathbf{k}}=\mathbf{k}^{2} / 2 m$. In particular, taking the state to be $|\psi\rangle=2^{-1 / 2}\left(\left|\mathbf{q}_{1}\right\rangle+\left|\mathbf{q}_{2}\right\rangle\right)$, which is the non-relativistic analog of the state $|\psi\rangle$ in Eq. (53), we find

$$
\langle\psi|n| \psi\rangle=V^{-1}\left\{1+\cos \left[\left(E_{\mathbf{q}_{1}}-E_{\mathbf{q}_{2}}\right) t-\left(\mathbf{q}_{1}-\mathbf{q}_{2}\right) \mathbf{x}\right]\right\} .
$$

Contrary to the relativistic particle density (53), the non-relativistic particle density (223) is non-negative.

\section{References}

[1] S. A. Fulling, Phys. Rev. D7, 2850 (1973).

[2] N. D. Birrell and P. C. W. Davies, Quantum Fields in Curved Space (Cambridge Press, NY, 1982).

[3] P. C. W. Davies, in Quantum Theory of Gravity, ed. S. M. Christensen (Adam Hilger Ltd, Bristol, 1984).

[4] W. G. Unruh, Phys. Rev. D14, 870 (1976). 
[5] B. S. DeWitt, in General Relativity: An Einstein Centenary Survey, eds. S. W. Hawking and W. Israel (Cambridge University Press, 1979).

[6] L. Parker, Phys. Rev. 183, 1057 (1969).

[7] S. W. Hawking, Commun. Math. Phys. 43, 199 (1975).

[8] W. G. Unruh and R. M. Wald, Phys. Rev. D29, 1047 (1984).

[9] D. W. Sciama, P. Candelas and D. Deutsch, Adv. Phys. 30, 327 (1981).

[10] C. Stephens, Ann. Phys. 193, 255 (1989).

[11] R. Parentani and R. Brout, Nucl. Phys. B388, 474 (1992).

[12] R. Brout, S. Massar, R. Parentani and Ph. Spindel, Phys. Rep. 260, 329 (1995).

[13] T. Padmanabhan, Phys. Rev. Lett. 64, 2471 (1990).

[14] H. Nikolić, Phys. Lett. B527, 119 (2002); Erratum-ibid. B529, 265 (2002).

[15] J. Schwinger, Phys. Rev. 82, 664 (1951).

[16] B. S. DeWitt, Phys. Rep. 19, 295 (1975).

[17] Y. Aharonov and D. Bohm, Phys. Rev. 122, 1649 (1961).

[18] L. H. Ford, Lectures given at the IX André Swieca Summer School, Campos dos Jordão, SP, Brazil, February 1997, and at Soochow University, Taipei, Taiwan, June 1997; grqc/9707062].

[19] J. D. Bjorken and S. D. Drell, Relativistic Quantum Fields (McGraw-Hill, New York, 1965).

[20] D. Marolf and C. Rovelli, Phys. Rev. D66, 023510 (2002).

[21] H. Nikolić, Mod. Phys. Lett. A16, 579 (2001).

[22] T. S. Bunch and L. Parker, Phys. Rev. D20, 2499 (1979).

[23] L. H. Ryder, Quantum Field Theory (Cambridge University Press, Cambridge, 1984).

[24] R. M. Wald, Quantum Field Theory in Curved Spacetime and Black Hole Thermodynamics (The University of Chicago Press, Chicago, 1994).

[25] V. A. Belinski, Phys. Lett. A209, 13 (1995).

[26] H. Nikolić, hep-th/0103053.

[27] H. Nikolić, hep-th/0103251.

[28] H. Nikolić, hep-ph/0105176.

[29] K. Srinivasan and T. Padmanabhan, gr-qc/9812087.

[30] M. Nouri-Zonoz and T. Padmanabhan, gr-qc/9812088.

[31] S. Weinberg, Gravitation and Cosmology (John Wiley \& Sons, NY, 1972). 
[32] A. Z. Capri and S. M. Roy, Mod. Phys. Lett. A7, 2317 (1992).

[33] A. Z. Capri and S. M. Roy, Int. J. Mod. Phys. A9, 1239 (1994).

[34] K. Melnikov and M. Weinstein, hep-th/0109201.

[35] C. A. Manogue, Ann. Phys. 181, 261 (1988).

[36] N. C. Tsamis, Class. Quant. Grav. 18, 83 (2001).

[37] L. I. Schiff, Quantum Mechanics (McGraw-Hill, Singapore, 1968).

[38] S. S. Schweber, An Introduction to Relativistic Quantum Field Theory (Harper \& Row, New York, 1961). 